\title{
Pacific
}

Journal of

Mathematics

\section{PARTIALLY MEASURABLE SETS IN MEASURE SPACES}

\section{MAX SHIFFMAN}




\section{Partially Measurable Sets in Measur Spaces}

\section{MaX SHIFFMAN}

1. Introduction. This article is concerned with any sets, whether measurable or not, in a general measure space (a general measure space is briefly defined in the article). A set $S$ has an interior measure $m_{i}(S)$ and an exterior measure $m_{e}(S)$, where $0 \leq m_{i}(S) \leq$ $m_{e}(S)$. Consider two disjoint sets $S_{1}$ and $S_{2}$, i.e. $S_{1} \cap S_{2}=\emptyset$, and their union set $S_{1} \cup S_{2}$. There are the six non-negative quantities $m_{i}(S)$ and $m_{e}(S)$, for $S=S_{1}, S_{2}$, and $S_{1} \cup S_{2}$. It is well-known that $m_{e}(S)$ is subadditive, i.e. $m_{e}\left(S_{1} \cup S_{2}\right) \leq m_{e}\left(S_{1}\right)+m_{e}\left(S_{2}\right)$, and $m_{i}(S)$ is superadditive, i.e. $m_{i}\left(S_{1} \cup S_{2}\right) \geq m_{i}\left(S_{1}\right)+m_{i}\left(S_{2}\right)$. The present article obtains more inequalities on the six quantities $m_{i}(S)$ and $m_{e}(S)$ for $S=S_{1}, S_{2}$, and $S_{1} \cup S_{2}$; and indeed obtains a specific collection of six linear inequalities. One of these six inequalities states that the average measure $\frac{1}{2}\left(m_{i}(S)+m_{e}(S)\right)$ is subadditive. Also, average measure is countably subadditive. Further, if the general measure space satisfies a certain two conditions, it is shown that these six inequalities form a complete collection of independent inequalities, valid for every pair of disjoint sets $S_{1}, S_{2}$. These two condition on the measure space do hold for the usual measure spaces.

Previous articles of the author considered the same matters for Lebesgue measure on the real number line or in Euclidean $n$-dimensional space. These are listed as [1] and [2] in the References at the end of the present article. The present article extends these results to a general measure space, subject to some limitations.

The inequalities on $m_{e}(S)$ and $m_{i}(S)$, for $S=S_{1}, S_{2}$, and $\left(S_{1} \cup\right.$ $S_{2}$ ), where $S_{1} \cap S_{2}=\emptyset$, are stated in Theorems 2 and 4 below, in $\S 3$. Furthermore, if the measure space satisfies two conditions, the partitionable condition and the basis condition, these inequalities are shown to be a complete collection of inequalities which are valid for every pair of disjoint sets contained in a measurable set $M$. 
This is stated in Theorem 8 in $\S 6$. The partitionable condition on a measure space is discussed in $\S 4$, and the basis condition in $\S 5$. These conditions do hold for Lebesgue or Borel measure, and in the usual measure spaces. The above results, without specifying the two conditions, were obtained in the author's previous articles cited above when the measure space is Lebesgue or Borel measure on the real number line or in Euclidean $n$-dimensional space.

A set $S$ for which $m_{i}(S)<m_{e}(S)$ has been called a non-measurable set. In the introduction to my previous article [2] in $\mathrm{Pa}$ cific Journal of Mathematics, it is stated that a non-measurable set $S$ might be more appropriately called a partially measurable set since one does have some information concerning the measure of $S$, namely that its "measure" is something between $m_{i}(S)$ and $m_{e}(S)$ inclusive. In the present article, I am indeed calling such a set partially measurable, as indicated in the title of the present article. In a broad sense of measure, if one is thinking of applications, not only in mathematics, an exact measurement might not be available for some process or subject. But a lower value and an upper value might be available, like interior measure and exterior measure. Or one might be concerned with estimates rather than measurements. Finding properties of the lower and upper values would be of interest.

Further results concerning partially measurable sets are obtained in the present article, including some interesting partially measurable sets. These latter are obtained in $\S 5$ and $\S 7$, and in particular in Theorems 7 and 9. Also, if one has two disjoint sets contained in a partially measurable set $S$, instead of in a measurable set $M$, a complete collection of inequalities is obtained in $\S 3$ and $\S 7$, stated in Theorems 4 and 10. These hold as well for Lebesgue or Borel measure on the real number line or in Euclidean space, and is a supplement to the previous articles cited above. It should also be stated that the present article, as well as the previous articles cited above, makes use of transfinite induction and the axiom of choice in set theory, but does not make use of the continuum hypothesis of set theory (which is that $2^{\chi_{0}}=\chi_{1}$ ).

2. Measure Spaces. The definition and properties of set theory and of measure spaces are fairly standard. For the sake of definiteness and generality, the definition and properties of a general 
measure space, as used in the present article, will be stated briefly here.

A general measure space, written $(X, \mathcal{M}, m)$, is a non-empty set $X$ of elements, which are called "points"; and a non-empty collection $\mathcal{M}$ of sets of points in $X$ which form a countable ring of sets; and a measure function $m(M)$ defined for every set $M$ in the collection $\mathcal{M}$, where $m(M)$ is a non-negative real number or $\infty$, and the measure function $m$ is required to be countably additive, and $m(\emptyset)=0$ where $\emptyset$ is the empty set.

The terms countable ring and countably additive are defined immediately below. A non-empty collection $\mathcal{M}$ of subsets of $X$ is called a ring if, whenever $M_{1}$ and $M_{2}$ are both members of the collection $\mathcal{M}$, written $M_{1} \in \mathcal{M}$ and $M_{2} \in \mathcal{M}$, then $\left(M_{1} \cup M_{2}\right) \in \mathcal{M}$ and $\left(M_{1}-M_{2}\right) \in \mathcal{M}$, where $\cup$ is the symbol for the set-theoretic sum (or union) of sets, and - in $M_{1}-M_{2}$ is the symbol for the set-theoretic difference of sets. Note that the empty set $\emptyset$ is in $M$, since $\emptyset=M-M$ for any $M$ in $\mathcal{M}$. It follows that $\left(M_{1} \cap M_{2}\right) \in \mathcal{M}$, where $\cap$ is the symbol for set-theoretic product (or intersection) of sets, since $\left(M_{1} \cap M_{2}\right)=M_{1}-\left(M_{1}-M_{2}\right)$, noting that $M_{1}-M_{2}=$ $M_{1}-\left(M_{1} \cap M_{2}\right)$. Consequently, $\left(\bigcup_{j=1}^{n} M_{j}\right) \in \mathcal{M}$ and $\left(\bigcap_{j=1}^{n} M_{j}\right) \in \mathcal{M}$ if $M_{j} \in \mathcal{M}$ for every $j=1,2, \ldots, n$. A non-empty collection $\mathcal{M}$ of subsets of $X$ is called a countable ring if $\mathcal{M}$ is a ring and if, whenever a countable infinity $M_{j}, j=1,2, \ldots, n, \ldots$, of sets are all in $\mathcal{M}$, then $\left(\bigcup_{j=1}^{\infty} M_{j}\right) \in \mathcal{M}$. It follows that $\left(\bigcap_{j=1}^{\infty} M_{j}\right) \in \mathcal{M}$, since $\left(\bigcap_{j=1}^{\infty} M_{j}\right)=M_{1}-\bigcup_{j=1}^{\infty}\left(M_{1}-M_{j}\right)$

The non-negative set function $m(M)$, defined for all sets $M$ in $\mathcal{M}$, is said to be additive if, whenever $M_{1} \in \mathcal{M}, M_{2} \in \mathcal{M}$, and $\left(M_{1} \cap M_{2}\right)=\emptyset$ (which is described by the words " $M_{1}$ and $M_{2}$ are disjoint sets"), then $m\left(M_{1} \cup M_{2}\right)=m\left(M_{1}\right)+m\left(M_{2}\right)$. The nonnegative set function $m(M)$, defined over the countable ring $\mathcal{M}$, is said to be countably additive if it is additive and if, whenever $M_{j}$ for $j=1,2, \ldots, n, \ldots$, are a countable infinity of mutually disjoint sets in $\mathcal{M}$, i.e., $\left(M_{j_{1}} \cap M_{j_{2}}\right)=\emptyset$ for every $j_{1}$ and $j_{2}$ with $j_{1} \neq j_{2}$, then 
$m\left(\bigcup_{j=1}^{\infty} M_{j}\right)=\sum_{j=1}^{\infty} m\left(M_{j}\right)$. Note that the values of $m(M)$ for all $M$ in $\mathcal{M}$ lie in the extended non-negative real number system, i.e., all non-negative real numbers and $\infty$. Incidentally, note that if $M_{1}, M_{2}$ are both in $\mathcal{M}$ and $M_{1} \subset M_{2}$ ( $\subset$ is the symbol for set-theoretic inclusion, and $\supset$ is the symbol for set-theoretic containment), then $m\left(M_{1}\right) \leq m\left(M_{2}\right)$, since $M_{2}=M_{1} \cup\left(M_{2}-M_{1}\right)$ and $M_{1} \cap\left(M_{2}-\right.$ $\left.M_{1}\right)=\emptyset$, so that $m\left(M_{2}\right)=m\left(M_{1}\right)+m\left(M_{2}-M_{1}\right) \geq m\left(M_{1}\right)$; and if $m\left(M_{1}\right)<\infty$ then $m\left(M_{2}-M_{1}\right)=m\left(M_{2}\right)-m\left(M_{1}\right)$. It also follows that if there is a set $M \in \mathcal{M}$ with $m(M)<\infty$, then $m(\emptyset)=0$ since $\emptyset=M-M$ and $m(\emptyset)=m(M)-m(M)=0$. Even if there is no such $M \in \mathcal{M}$, it is supposed that $m(\emptyset)=0$.

The above gives the definition of a measure space $(X, \mathcal{M}, m)$ as used in this article. Note that the above definition of a general measure space does not assume that the set $X$ is in $\mathcal{M}$. If $X \notin$ $\mathcal{M}$, the measure space $(X, \mathcal{M}, m)$ can be extended, if it is desired, to a measure space $\left(X, \mathcal{M}^{*}, m^{*}\right)$ in which the countable ring $\mathcal{M}^{*}$ includes the set $X$ as well as all of $\mathcal{M}$, and $m^{*}(M)=m(M)$ for all $M \in \mathcal{M}$. This is discussed near the end of $\S 6$ herein. Also note that the definition of a general measure space does not assume the "countability condition for a measure space", which is stated and discussed in the latter part of $\S 6$ herein, and this matter does not enter prior to that, nor in $\S 7$. And the definition of a general measure space does not assume that it is a complete measure space, i.e. that every subset of a set $M$ in $\mathcal{M}$ with $m(M)=0$ also is in $\mathcal{M}$ and has $m$-measure 0 . The measure space can be extended, if desired, by completing it.

Consider a general measure space $(X, \mathcal{M}, m)$. For any set $S$ of points in $X$, the interior measure $m_{i}(S)$ and exterior measure $m_{e}(S)$ can be defined (for $m_{e}(S)$, it is required that there be a set $M \in \mathcal{M}$ for which $S \subset M)$. The interior measure of $S$ (also called inner measure of $S$ ), written $m_{i}(S)$, is defined as usual by

(2.1) $m_{i}(S)=\sup _{M \subset S} m(M)$, for all $M \in \mathcal{M}$ with $M \subset S$,

where $\sup _{M \subset S} m(M)$ is the supremum, or least upper bound, of all the numbers $m(M)$ with $M \in \mathcal{M}$ and $M \subset S$. There is at least one such $M$, namely $\emptyset$ for which $m(\emptyset)=0$. As is well-known, shown 
from (2.1),

$$
\begin{cases}m_{i}(S) \geq 0 ; \text { and if } S_{1} \subset S_{2}, \text { then } & m_{i}\left(S_{1}\right) \leq m_{i}\left(S_{2}\right) ; \\ \text { and if } S \in \mathcal{M}, \text { then } & m_{i}(S)=m(S) ; \\ \text { and if } S_{j}, j=1,2, \ldots \text { to } n \text { or } & \\ \text { to } \infty, \text { are mutually disjoint, then } & m_{i}\left(\bigcup_{j} S_{j}\right) \\ & \geq \sum_{j} m_{i}\left(S_{j}\right) .\end{cases}
$$

The last in (2.2) states that the set function $m$ is superadditive, and indeed is countably superadditive, for disjoint sets. Also,

$$
\text { there is a set } A \subset S \text { for which }
$$

$$
A \in \mathcal{M} \text { and } m(A)=m_{i}(S) \text {. }
$$

For the exterior measure $m_{e}(S)$ of a set $S$ (also called outer measure of $S$ ), suppose that there is a set $M \in \mathcal{M}$ for which $S \subset M$. (If $X \in \mathcal{M}$, this is satisfied for every $S$.) The exterior measure $m_{e}(S)$ is defined as usual by

$$
m_{e}(S)=\inf _{M \supset S} m(M), \text { for all } M \in \mathcal{M} \text { with } M \supset S,
$$

where $\inf _{M \supset S}$ is the infimum, or greatest lower bound, of all the numbers $m(M)$ with $M \supset S$ and $M \in \mathcal{M}$. As is well-known, shown from (2.4)

$$
\begin{cases}m_{e}(S) \geq m_{i}(S) \geq 0 ; & \\ \text { and if } S_{1} \subset S_{2}, \text { then } & m_{e}\left(S_{1}\right) \leq m_{e}\left(S_{2}\right) ; \\ \text { and if } S \in \mathcal{M}, \text { then } & m_{e}(S)=m(S) ; \\ \text { and if } S_{j}, j=1,2, \ldots, \text { to } n & \\ \text { or to } \infty, \text { are a countable } & \\ \text { aumber of sets, then } & m_{e}\left(\bigcup_{j} S_{j}\right) \leq \sum_{j} m_{e}\left(S_{j}\right) .\end{cases}
$$

The last in (2.5) states that the set function $m_{e}(S)$ is subadditive, and indeed is countably subadditive. Also,

(2.6) there is a set $B \in \mathcal{M}$ for which $S \subset B \subset M$ and

$$
m(B)=m_{e}(S)
$$


The above in (2.4), (2.5), (2.6) assumed that there was a set $M \in \mathcal{M}$ such that the sets $S$ and $S_{1}, S_{2}$, and $S_{j}$ are all contained in $M$.

Note that, in the general measure space $(X, \mathcal{M}, m)$, the interior measure $m_{i}(S)$ is defined for every set $S$ in $X$, but the exterior measure $m_{e}(S)$ is defined for a set $S$ only if there is a set $M$ in $\mathcal{M}$ for which $S \subset M$. Whenever $m_{e}(S)$ is used in the present article it is assumed that there is a set $M \in \mathcal{M}$ with $S \subset M$. If $X \notin M$, there are sets $S$ with no such $M$, for example the set $X$ itself. If $X \notin \mathcal{M}$, the measure space $(X, \mathcal{M}, m)$ can be extended if desired to a measure space $\left(X, \mathcal{M}^{*}, m^{*}\right)$ in which $X \in \mathcal{M}^{*}$, as discussed near the end of $\S 6$ herein.

An example of a measure space $(X, \mathcal{M}, m)$ is Lebesgue measure on the real number line or in Euclidean $n$-dimensional space (or Borel measure). In this case, $X$ is the set of points on the real number line or in Euclidean space, and $\mathcal{M}$ is the collection of Lebesgue (or Borel) measurable sets, and the measure function $m(M)$ is the Lebesgue (or Borel) measure of $M$. Then for any set $S$ the interior measure $m_{i}(S)$ and exterior measure $m_{e}(S)$ are the usual Lebesgue interior and exterior measures of $S$.

Consider now a general measure space $(X, \mathcal{M}, m)$. There are some lemmas concerning the interior and exterior measures, $m_{i}(S)$ and $m_{e}(S)$.

Lemma 1. Suppose $M^{j} \in \mathcal{M}, j=1,2, \ldots$ to $n$ or $\infty$, are a countable number of mutually disjoint sets in $\mathcal{M}$. Then for any set $S$,

$$
\left\{\begin{array}{l}
m_{i}(S)=\sum_{j} m_{i}\left(M^{j} \cap S\right)+m_{i}\left(\left(X-\bigcup_{j} M^{j}\right) \cap S\right) \\
m_{e}(S)=\sum_{j} m_{e}\left(M^{j} \cap S\right)+m_{e}\left(\left(X-\bigcup_{j} M^{j}\right) \cap S\right)
\end{array}\right.
$$

where in the second line of (2.7), $S \subset M$ for some $M \in \mathcal{M}$.

Proof. Select a set $A \in \mathcal{M}$ with $A \subset S$ and $m(A)=m_{i}(S)$. Then $\left(M^{j} \cap A\right) \subset\left(M^{j} \cap S\right)$ so that $m\left(M^{j} \cap A\right) \leq m_{i}\left(M^{j} \cap S\right)$; and

$$
\left(X-\bigcup_{j} M^{j}\right) \cap A=\left(A-\bigcup_{j}\left(M^{j} \cap A\right)\right) \in \mathcal{M}, \text { and }
$$




$$
\begin{gathered}
\left(\left(X-\bigcup_{j} M^{j}\right) \cap A\right) \subset\left(\left(X-\bigcup_{j} M^{j}\right) \cap S\right) \text { so that } \\
m\left(A-\bigcup_{j}\left(M^{j} \cap A\right)\right) \leq m_{i}\left(\left(X-\bigcup_{j} M^{j}\right) \cap S\right) . \text { Since } \\
A=\left(\bigcup_{j}\left(M^{j} \cap A\right)\right) \cup\left(A-\bigcup_{j}\left(M^{j} \cap A\right)\right), \text { one has } \\
m_{i}(S)=m_{(A)}=\left(\sum_{j} m_{i}\left(M^{j} \cap A\right)\right)+m\left(A-\bigcup_{j}\left(M^{j} \cap A\right)\right) \\
\leq\left(\sum_{j} m_{i}\left(M^{j} \cap S\right)\right)+m_{i}\left(X-\bigcup_{j}\left(M^{j} \cap S\right)\right) .
\end{gathered}
$$

But interior measure $m_{i}$ is countably superadditive for disjoint sets, as in (2.2), which is the reverse inequality for $m_{i}(S)$, so that the first equality of (2.7) is established.

Select a set $B \in \mathcal{M}$ with $S \subset B \subset M$ and $m(B)=m_{e}(S)$. Then $\left(M^{j} \cap S\right) \subset\left(M^{j} \cap B\right)$ so that $m\left(M^{j} \cap B\right) \geq m_{e}\left(M^{j} \cap S\right)$; and $\left(\left(X-\bigcup_{j} M^{j}\right) \cap S\right) \subset\left(\left(X-\bigcup_{j} M^{j}\right) \cap B\right)=\left(B-\bigcup_{j}\left(M^{j} \cap B\right)\right) \in \mathcal{M}$ so that $m\left(B-\bigcup_{j}\left(M^{j} \cap B\right)\right) \geq m_{e}\left(\left(X-\bigcup_{j} M^{j}\right) \cap S\right)$. Since $B=\bigcup_{j}\left(M^{j} \cap B\right) \cup\left(B-\bigcup_{j}\left(M^{j} \cap B\right)\right)$ one has

$$
\begin{aligned}
m_{e}(S)=m(B) & =\left(\sum_{j} m\left(M^{j} \cap B\right)\right)+m\left(B-\bigcup_{j}\left(M^{j} \cap B\right)\right) \\
& \geq\left(\sum_{j} m_{e}\left(M^{j} \cap S\right)\right)+m_{e}\left(\left(X-\bigcup_{j} M^{j}\right) \cap S\right) .
\end{aligned}
$$

But exterior measure $m_{e}$ is countably subadditive, as in (2.5), which is the reverse inequality for $m_{e}(S)$, so that the second equality of (2.7) is established. Lemma 1 is proved.

Lemma 1 is an extension to a general measure space of Lemma 1 in the author's article, Measure-theoretic Properties of Non-measurable Sets, Pacific J. Math. 138(1989), 357-389, which is listed in the Bibliography at the end of the present article as [2]. This lemma is on p.359, proof on p.360, of [2]; and Lemma 1 above, where 
$S=\bigcup_{\nu} S_{\nu}$, is an extension to $(X, \mathcal{M}, m)$ since $X$ need not be in $\mathcal{M}$. The article Lemma 1 concerns Lebesgue measure on the real number line or in Euclidean $n$-dimensional space.

Also, Lemmas 2 and 3 and their proofs, on pages 360, 361 of [2], hold in a general measure space $(X, \mathcal{M}, m)$. Just replace the word "measurable" and words "measurable set" by the words "in $\mathcal{M}$ " and "set in $\mathcal{M}$ " respectively. For the statements and proofs use only the countable ring and countable additivity properties of Lebesgue measure, and these hold in $(X, \mathcal{M}, m)$. Also, formulas (2.1) on the page 360 of [2] hold; just replace the sentence after formulas $(2.1)$ by the sentence: This is by Lemma 1 , since for the second formula of (2.1) $S$ is contained in the set $(M-(L \cap M))$, which is in $\mathcal{M}$ and is disjoint from $L$, where $S \subset M \in \mathcal{M}$. Note again that throughout the present article, if $m_{e}(S)$ is used, then it is supposed that there is a set $M$ in $\mathcal{M}$ such that $S \subset M$. Lemma 2 is stated below, as Lemma 2 , since it is used frequently in later sections of the present article; its proof is on pages 360,361 of [2].

Lemma 2. Suppose that $\left(S_{1} \cup S_{2}\right)$ is in $\mathcal{M}$, where $S_{1}$ and $S_{2}$ are disjoint sets, $S_{1} \cap S_{2}=\emptyset$. Then

$$
m\left(S_{1} \cup S_{2}\right)=m_{i}\left(S_{1}\right)+m_{e}\left(S_{2}\right)=m_{e}\left(S_{1}\right)+m_{i}\left(S_{2}\right) .
$$

Theorem 4 on page 372 of [2] also holds in a general measure space $(X, \mathcal{M}, m)$. The inequalities for $m_{e}\left(S_{1} \cup S_{2}\right)$ in that theorem are proved in the first paragraph of the proof on page 372 of [2], where $L$ and $B_{1}$ are in $\mathcal{M}$. The inequalities for $m_{i}\left(S_{1} \cup S_{2}\right)$ in that theorem can be proved similarly, instead of the proof that is given on pages 372,373 of [2] (which involves the functionals $d_{i}$ and $d_{e}$ and $d_{i} \leq d_{e}$, to be taken up later in the present article), as follows. Pick a set $K \in \mathcal{M}$ with $K \subset\left(S_{1} \cup S_{2}\right)$ and $m(K)=m_{i}\left(S_{1} \cup S_{2}\right)$, and a set $A_{2} \in \mathcal{M}$ with $A_{2} \supset S_{2}$ and $m\left(A_{2}\right)=m_{e}\left(S_{2}\right)$. Then $\left(K-\left(K \cap A_{2}\right)\right) \subset S_{1}$, since a point in $\left(K-\left(K \cap A_{2}\right)\right)$ is in $K$ and not in $A_{2}$, and so not in $S_{2}$, and therefore is in $S_{1}$ since $K \subset\left(S_{1} \cup S_{2}\right)$. Thus, $m\left(K-\left(K \cap A_{2}\right)\right) \leq m_{i}\left(S_{1}\right)$; also $m\left(K \cap A_{2}\right) \leq m\left(A_{2}\right)=$ 
$m_{e}\left(S_{2}\right)$. Now

$$
\begin{aligned}
K & =\left(K-\left(K \cap A_{2}\right)\right) \cup\left(K \cap A_{2}\right) \quad \text { so that } \\
m_{i}\left(S_{1} \cup S_{2}\right) & =m(K)=m\left(K-\left(K \cap A_{2}\right)\right)+m\left(K \cap A_{2}\right) \\
& \leq m_{i}\left(S_{1}\right)+m_{e}\left(S_{2}\right) .
\end{aligned}
$$

This is one of the inequalities for $m_{i}\left(S_{1} \cup S_{2}\right)$ in theorem 4 of [2], and the other inequality is obtained by interchanging the roles of $S_{1}$ and $S_{2}$. Theorem 4 on page 372 of [2] is thus proved for a general measure space $(X, \mathcal{M}, m)$.

It will be important to consider those sets $\widetilde{M}$ in $\mathcal{M}$ for which

$$
\begin{cases}\text { either } & m(\widetilde{M})<\infty \\ \text { or } & m(\widetilde{M})=\infty \text { and } \widetilde{M}=\bigcup_{j=1}^{\infty} M^{j} \text { where } \\ & M^{j} \in \mathcal{M} \text { and } m\left(M^{j}\right)<\infty \text { for all } j .\end{cases}
$$

In (2.8), it suffices that $\widetilde{M} \subset\left(\bigcup_{j=1}^{\infty} M^{j}\right)$ since then $\widetilde{M}=\bigcup_{j=1}^{\infty}\left(\widetilde{M} \cap M^{j}\right)$. In case $m(\widetilde{M})=\infty$ and $\widetilde{M}=\bigcup_{j=1}^{\infty} M^{j}$ as in $(2.8)$, then $\widetilde{M}=\bigcup_{j=1}^{\infty}\left(M^{j}-\right.$ $\left.\bigcup_{k=1}^{j-1} M^{k}\right)$, where for $j=1, \bigcup_{k=1}^{0} M^{k}$ means $\emptyset$. The sets $\widetilde{M}^{j}=M^{j}-$ $k=1$ $j-1$

$\bigcup M^{k}$ for all $j=1,2, \ldots$ to $\infty$ are mutually disjoint since $k=1$

$$
\left(M^{j}-\bigcup_{k=1}^{j-1} M^{k}\right) \cap M^{n}=\emptyset \text { for } n<j
$$

and $\widetilde{M}^{j} \in \mathcal{M}$ with $m\left(\widetilde{M}^{j}\right) \leq m\left(M^{j}\right)<\infty$, and unite all the $\widetilde{M}^{j}$ for which $m\left(\widetilde{M}^{j}\right)=0$ together with an $\widetilde{M}^{j}$ for which $m\left(\widetilde{M}^{j}\right)>0$; and renumber the resulting j's from 1 to $\infty$. Thus, a set $\widetilde{M}$ which satisfies (2.8) and has $m(\widetilde{M})=\infty$ can be expressed as

$$
\begin{cases}\widetilde{M}=\bigcup_{j=1}^{\infty} \widetilde{M}^{j} & \text { where } \widetilde{M}^{j} \in \mathcal{M} \text { and } \\ \widetilde{M} & 0<m\left(\widetilde{M}^{j}\right)<\infty \text { for all } j, \text { and }\end{cases}
$$

$\widetilde{M}^{j_{1}} \cap \widetilde{M}^{j_{2}}=\emptyset \quad$ for all $j_{1}, j_{2}$ with $j_{1} \neq j_{2}$. 
The collection of all sets $\widetilde{M}$ in $\mathcal{M}$ which satisfy (2.8), will be designated by $\widetilde{\mathcal{M}}$ :

$$
\begin{aligned}
& \widetilde{\mathcal{M}}=\text { collection of all sets } \widetilde{M} \text { in } \mathcal{M} \text { which } \\
& \text { satisfy }(2.8) \text {, or the sentence below }(2.8) .
\end{aligned}
$$

Concerning the collection $\widetilde{\mathcal{M}}$, it is easily seen to be a countable ring. Also, $(M \cap \widetilde{M}) \in \widetilde{\mathcal{M}}$ for every $M \in \mathcal{M}, \widetilde{M} \in \widetilde{\mathcal{M}}$. Indeed, using the same measure $m(\widetilde{M})$ for all $\widetilde{M} \in \widetilde{\mathcal{M}}$ as in $(X, \mathcal{M}, m)$, one sees that $(X, \widetilde{\mathcal{M}}, m)$ is a measure space also. And note that if $S \subset \widetilde{M}_{1}$ for some $\widetilde{M}_{1} \in \widetilde{\mathcal{M}}$, then $m_{i}(S)$ and $m_{e}(S)$ obtained in $(X, \widetilde{\mathcal{M}}, m)$ have the same values as $m_{i}(S)$ and $m_{e}(S)$ obtained in $(X, \mathcal{M}, m)$. For, if $M \in \mathcal{M}$ and $M \subset S$ then $M \subset \widetilde{M}_{1}$ and $M=\left(M \cap \widetilde{M}_{1}\right) \in \widetilde{\mathcal{M}}$, so that $m_{i}(S)$ is the same. And if $M \in \mathcal{M}$ and $M \supset S$, then $(M \cap \widetilde{M}) \supset S$ for every $\widetilde{M} \in \widetilde{\mathcal{M}}$ with $\widetilde{M} \supset S$, and $m(M) \geq m(M \cap \widetilde{M})$ so that in taking the infimum of $m(M)$ one can limit oneself to sets $M \cap \widetilde{M}$, and these are in $\widetilde{\mathcal{M}}$; thus $m_{e}(S)$ is the same. Also, concerning $\widetilde{\mathcal{M}}$, there is Lemma 6 stated and proved in $\S 7$ of the present article. And in $\S 6$ of the present article, a condition is stated when $\widetilde{\mathcal{M}}=\mathcal{M}$.

\section{Functionals and Inequalities for Two Disjoint Sets.} Consider a general measure space $(X, \mathcal{M}, m)$, and consider any subsets $S$ of $X$. For two disjoint sets $S_{1}$ and $S_{2}$, several functionals of the pair $S_{1}, S_{2}$ will be defined. The notation and definitions of these functionals are the same as in the author's article, Measure-theoretic Properties of Non-measurable Sets, Pacific J. Math. 138(1989), 357389 , which is listed in the Bibliography at the end of the present article as [2]. Article [2] concerns Lebesgue measure on the real number line or in Euclidean $n$-dimensional space, and the results in $[2]$ are being generalized to a general measure space $(X, \mathcal{M}, m)$ in the present article. The definitions will first be given immediately below for the case that

$$
m_{e}\left(S_{1} \cup S_{2}\right)<\infty \text {, where }\left(S_{1} \cap S_{2}\right)=\emptyset \text {. }
$$

The functionals are written as $d_{i}\left(S_{1}, S_{2}\right), d_{e}\left(S_{1}, S_{2}\right), g_{1}\left(S_{1}, S_{2}\right)$, 
$g_{2}\left(S_{1}, S_{2}\right)$, and $h\left(S_{1}, S_{2}\right)$. Their definitions in the case (3.1) are:

$$
\left\{\begin{aligned}
d_{i}\left(S_{1}, S_{2}\right) & =m_{i}\left(S_{1} \cup S_{2}\right)-m_{i}\left(S_{1}\right)-m_{i}\left(S_{2}\right), \\
d_{e}\left(S_{1}, S_{2}\right) & =m_{e}\left(S_{1}\right)+m_{e}\left(S_{2}\right)-m_{e}\left(S_{1} \cup S_{2}\right), \\
g_{1}\left(S_{1}, S_{2}\right)= & m_{e}\left(S_{1} \cup S_{2}\right)-m_{i}\left(S_{1}\right)-m_{e}\left(S_{2}\right), \\
g_{2}\left(S_{1}, S_{2}\right)= & m_{e}\left(S_{1} \cup S_{2}\right)-m_{i}\left(S_{2}\right)-m_{e}\left(S_{1}\right), \\
h\left(S_{1}, S_{2}\right)= & m_{i}\left(S_{1}\right)+m_{e}\left(S_{1}\right)+m_{i}\left(S_{2}\right)+m_{e}\left(S_{2}\right) \\
& -m_{i}\left(S_{1} \cup S_{2}\right)-m_{e}\left(S_{1} \cup S_{2}\right) .
\end{aligned}\right.
$$

Another form for the last equation in (3.2) is

$$
h\left(S_{1}, S_{2}\right)=d_{e}\left(S_{1}, S_{2}\right)-d_{i}\left(S_{1}, S_{2}\right)
$$

which follows from the first two equations in (3.2). These functionals will be shown to be non-negative in value, as in [2]. That the first two functionals in $(3.2)$, i.e. $d_{i}\left(S_{1}, S_{2}\right)$ and $d_{e}\left(S_{1}, S_{2}\right)$, are $\geq 0$ is well-known, expressing the superadditivity of interior measure and subadditivity of exterior measure. Note that also $h\left(S_{1}, S_{2}\right)$ is stated to be $\geq 0$, where $h\left(S_{1}, S_{2}\right)$ is given in (3.3).

Let $f\left(S_{1}, S_{2}\right)$ be any one of the functionals $d_{i}\left(S_{1}, S_{2}\right), d_{e}\left(S_{1}, S_{2}\right)$, $g_{1}\left(S_{1}, S_{2}\right), g_{2}\left(S_{1}, S_{2}\right), h\left(S_{1}, S_{2}\right)$. There is the following theorem.

THEOREM 1. Consider two disjoint sets $S_{1}$ and $S_{2}$ which are contained in $\widetilde{M}$ for some $\widetilde{M}$ in $\widetilde{\mathcal{M}}$, where $\widetilde{\mathcal{M}}$ is defined in (2.10):

(3.4) $\left(S_{1} \cup S_{2}\right) \subset \widetilde{M}$ for some $\widetilde{M} \in \widetilde{\mathcal{M}}$, where $\left(S_{1} \cap S_{2}\right)=\emptyset$.

Let $f\left(S_{,} S_{2}\right)$ be any one of the functionals $d_{i}\left(S_{1}, S_{2}\right), d_{e}\left(S_{1}, S_{2}\right)$, $g_{1}\left(S_{1}, S_{2}\right), g_{2}\left(S_{1}, S_{2}\right), h\left(S_{1}, S_{2}\right)$. Suppose that $L_{k}$, for $k=1,2, \ldots$ to $n$ or to $\infty$, are a countable number of mutually disjoint sets in $\mathcal{M}$. Then

$$
\begin{aligned}
f\left(S_{1}, S_{2}\right)= & \sum_{k} f\left(L_{k} \cap S_{1}, L_{k} \cap S_{2}\right) \\
& +f\left(\left(X-\bigcup_{k} L_{k}\right) \cap S_{1},\left(X-\bigcup_{k} L_{k}\right) \cap S_{2}\right) .
\end{aligned}
$$


(Note that $\left(X-\bigcup_{k} L_{k}\right)$ in $(3.5)$ can be replaced by $\left(\widetilde{M}-\bigcup_{k} L_{k}\right)$, and also if $\left(S_{1} \cup S_{2}\right) \subset\left(\bigcup_{k} L_{k}\right)$ then the last term in (3.5) is 0 since $f(\emptyset, \emptyset)=0$.)

Proof. The theorem will first be proved when $m_{e}\left(S_{1} \cup S_{2}\right)<\infty$. Given any two disjoint sets $S_{1}$ and $S_{2}$ such that $m_{e}\left(S_{1} \cup S_{2}\right)<\infty$, there is a set $M$ in $\mathcal{M}$ such that $\left(S_{1} \cup S_{2}\right) \subset M$ and $m(M)=m_{e}\left(S_{1} \cup\right.$ $\left.S_{2}\right)<\infty$, so that $M \in \widetilde{\mathcal{M}}$ by (2.8), and (3.4) holds. Consider any particular case for $f\left(S_{1}, S_{2}\right)$, for example $f\left(S_{1}, S_{2}\right)=g_{1}\left(S_{1}, S_{2}\right)$. By (3.2) applied to the disjoint sets $L_{k} \cap S_{1}, L_{k} \cap S_{2}$ one has $g_{1}\left(L_{k} \cap S_{1}, L_{k} \cap S_{2}\right)=m_{e}\left(L_{k} \cap\left(S_{1} \cup S_{2}\right)\right)-m_{i}\left(L_{k} \cap S_{1}\right)-m_{e}\left(L_{k} \cap S_{2}\right)$. since $\left(L_{k} \cap S_{1}\right) \cup\left(L_{k} \cap S_{2}\right)=L_{k} \cap\left(S_{1} \cup S_{2}\right)$, and summing for $k$ going from 1 to $n$ gives

$$
\begin{aligned}
\sum_{k=1}^{n} g_{1}\left(L_{k} \cap S_{1}, L_{k} \cap S_{2}\right)= & \sum_{k=1}^{n} m_{e}\left(L_{k} \cap\left(S_{1} \cup S_{2}\right)\right) \\
& -\sum_{k=1}^{n} m_{i}\left(L_{k} \cap S_{1}\right) \\
& -\sum_{k=1}^{n} m_{e}\left(L_{k} \cap S_{2}\right) .
\end{aligned}
$$

If the index $k$ in Theorem 1 runs from 1 to $\infty$, let $n \rightarrow \infty$ in (3.6), giving

$$
\begin{aligned}
\sum_{k=1}^{\infty} g_{1}\left(L_{k} \cap S_{1}, L_{k} \cap S_{2}\right)= & \sum_{k=1}^{\infty} m_{e}\left(L_{k} \cap\left(S_{1} \cup S_{2}\right)\right) \\
& -\sum_{k=1}^{\infty} m_{i}\left(L_{k} \cap S_{1}\right) \\
& -\sum_{k=1}^{\infty} m_{e}\left(L_{k} \cap S_{2}\right),
\end{aligned}
$$

since the quantities on the right-hand side are less than or equal to $m_{e}\left(S_{1} \cup S_{2}\right), m_{i}\left(S_{1}\right), m_{e}\left(S_{2}\right)$ respectively, by Lemma 1 . There is also

$$
\begin{aligned}
g_{1}\left(Y \cap S_{1}, Y \cap S_{2}\right)= & m_{e}\left(Y \cap\left(S_{1} \cup S_{2}\right)\right) \\
& -m_{i}\left(Y \cap S_{1}\right)-m_{e}\left(Y \cap S_{2}\right)
\end{aligned}
$$


where $Y=X-\bigcup_{k=1}^{n} L_{k}$ or $Y=X-\bigcup_{k=1}^{\infty} L_{k}$, according as $k$ runs from 1 to $n$ or 1 to $\infty$ in Theorem 1. Adding (3.8) to (3.6) or (3.7) accordingly yields, for the right-hand side of $(3.5)$ when $f\left(S_{1}, S_{2}\right)$ is $g_{1}\left(S_{1}, S_{2}\right)$, the value

$$
m_{e}\left(S_{1} \cup S_{2}\right)-m_{i}\left(S_{1}\right)-m_{e}\left(S_{2}\right),
$$

by Lemma 1 . The expression (3.9) is equal to $g_{1}\left(S_{1}, S_{2}\right)$ by (3.2). Thus, (3.5) is established when $f\left(S_{1}, S_{2}\right)$ is $g_{1}\left(S_{1}, S_{2}\right)$.

A similar proof holds for any of the other functionals $d_{i}\left(S_{1}, S_{2}\right)$, $d_{e}\left(S_{1}, S_{2}\right), g_{2}\left(S_{1}, S_{2}\right), h\left(S_{1}, S_{2}\right)$ in (3.2). Theorem 1 is therefore proved for the case that $m_{e}\left(S_{1} \cup S_{2}\right)<\infty$.

Incidentally, Theorem 1 above, for the case that $m_{e}\left(S_{1} \cup S_{2}\right)<\infty$ and $f\left(S_{1}, S_{2}\right)$ is $d_{i}\left(S_{1}, S_{2}\right)$ and $d_{e}\left(S_{1}, S_{2}\right)$, is stated and proved in [2], on page 362 of [2], as Lemma 4 on page 362, where $S_{1}=$ $\cup S_{1}^{\nu}, S_{2}=\cup S_{2}^{\nu}$, and $\left(S_{1}^{\nu} \cup S_{1}^{\nu}\right) \subset L^{\underline{\nu}}$, the $L^{\underline{\nu}}$ being disjoint measurable sets. (There is a misprint on page 365 of [2], in formula (3.5) there; it should read $\sum_{\underline{\nu}}$, not $\sum_{\underline{\mu}}$.) It is stated in later pages of [2] that a similar proof holds for $g_{1}\left(S_{1}, S_{2}\right), g_{2}\left(S_{1}, S_{2}\right)$ and $h\left(S_{1}, S_{2}\right)$.

Still for the case that $m_{e}\left(S_{1} \cup S_{2}\right)<\infty$, the quantities $d_{i}\left(S_{1}, S_{2}\right)$ and $d_{e}\left(S_{1}, S_{2}\right)$ are non-negative, since they state the superadditivity of interior measure respectively. And also $g_{1}\left(S_{1}, S_{2}\right) \geq 0$ and $g_{2}\left(S_{1}, S_{2}\right) \geq 0$, by Theorem 4 on page 372 of [2] (using the inequalities for $m_{e}\left(S_{1} \cup S_{2}\right)$ in that Theorem 4), which holds in a general measure space $(X, \mathcal{M}, m)$, as stated and proved in the preceding section, $\S 2$ of the present article.

Also, the functional $h\left(S_{1}, S_{2}\right)$ is non-negative, stated in the form

$$
d_{i}\left(S_{1}, S_{2}\right) \leq d_{e}\left(S_{1}, S_{2}\right),
$$

The inequality (3.10) is stated as a theorem on page 367 of [2], and proved on pages 367-369. The same proof holds in the general measure space $(X, \mathcal{M}, m)$, when $m_{e}\left(S_{1} \cup S_{2}\right)<\infty$, replacing the words "measurable set" by "set in $\mathcal{M}$ ", and "(the entire space $\left.B_{1}\right)$ " and "(the entire space - $\left.B_{2}\right)$ " by " $\left(M-B_{1}\right)$ " and " $\left(M-B_{2}\right)$ " respectively, where $M \in \mathcal{M}$ and $\left(S_{1} \cup S_{2}\right) \subset M$ with $m(M)=$ $m_{e}\left(S_{1} \cup S_{2}\right)<\infty$; and also "bounded set" by "set with finite exterior 
measure". Note that Lemma 4 in [2], referred to in the proof on page 367 of [2], is included in Theorem 1 of the present article, as stated two paragraphs above. Thus $(3.10)$ holds, so that $h\left(S_{1}, S_{2}\right)$ in (3.3) and (3.2) is non-negative.

Thus, all the functionals $d_{i}\left(S_{1}, S_{2}\right), d_{e}\left(S_{1}, S_{2}\right), g_{1}\left(S_{1}, S_{2}\right), g_{2}\left(S_{1}, S_{2}\right)$ and $h\left(S_{1}, S_{2}\right)$, defined in (3.2) (and also (3.3)), for the case that $m_{e}\left(S_{1} \cup S_{2}\right)<\infty$, are non-negative. Their definitions will now be given for two disjoint sets $S_{1}, S_{2}$, when

$$
\begin{aligned}
& m_{e}\left(S_{1} \cup S_{2}\right)=\infty \text { and } \\
& \left(S_{1} \cup S_{2}\right) \subset \widetilde{M} \text { for some } \widetilde{M} \in \widetilde{\mathcal{M}}
\end{aligned}
$$

where $\widetilde{\mathcal{M}}$ is defined in $(2.10)$. Then $m(\widetilde{M})=\infty$, and the set $\widetilde{M}$ can be expressed, by (2.9) as

$$
\begin{cases}\widetilde{M}=\bigcup_{j=1}^{\infty} M^{j}, & \text { where } M^{j} \in \widetilde{M} \text { and } m\left(M^{j}\right)<\infty \\ & \text { for all } j, \text { and } M^{j_{1}} \cap M^{j_{2}}=\emptyset \\ & \text { for all } j_{1}, j_{2} \text { with } j_{1} \neq j_{2} .\end{cases}
$$

Let $f\left(S_{1}, S_{2}\right)$ be any one of the functionals $d_{i}\left(S_{1}, S_{2}\right), d_{e}\left(S_{1}, S_{2}\right)$, $g_{1}\left(S_{1}, S_{2}\right), g_{2}\left(S_{1}, S_{2}\right)$ or $h\left(S_{1}, S_{2}\right)$, and define $f\left(S_{1}, S_{2}\right)$ by

$$
f\left(S_{1}, S_{2}\right)=\sum_{j=1}^{\infty} f\left(M^{j} \cap S_{1}, M^{j} \cap S_{2}\right)
$$

One has that $m_{e}\left(\left(M^{j} \cap S_{1} 0 \cup\left(M^{j} \cap S_{2}\right)\right) \leq m\left(M^{j}\right)<\infty\right.$, and the formula in (3.2) can be applied to obtain $f\left(M^{j} \cap S_{1}, M^{j} \cap S_{2}\right)$, which is finite and non-negative, and (3.13) yields $f\left(S_{1}, S_{2}\right)$. Note that $\infty$ is a possible value of $f\left(S_{1}, S_{2}\right)$. The values of $f\left(S_{1}, S_{2}\right)$ are in the extended non-negative real number system, including $\infty$ as well as non-negative real numbers.

Concerning $f\left(S_{1}, S_{2}\right)$ as defined in (3.12) and (3.13), if $\widetilde{M}=$ $\bigcup_{j=1}^{\infty} N^{j}$, where $N^{k} \in \mathcal{M}$ and $m\left(N^{k}\right)<\infty$ and the $N^{k}$ are mutually disjoint, is another expression for $M$ as in (3.12), then

$$
f\left(N^{k} \cap S_{1}, N^{k} \cap S_{2}\right)=\sum_{j=1}^{\infty} f\left(M^{j} \cap\left(N^{k} \cap S_{1}\right), M^{j} \cap\left(N^{k} \cap S_{2}\right)\right)
$$


by Theorem 1 already proved for the finite case since $m_{e}\left(\left(N^{k} \cap S_{1}\right) \cup\right.$ $\left.\left(N^{k} \cap S_{1}\right)\right) \leq m\left(N^{k}\right)<\infty$ and $\left(\left(N^{k} \cap S_{1}\right) \cup\left(N^{k} \cap S_{1}\right)\right) \subset\left(S_{1} \cup S_{2}\right) \subset$ $\widetilde{M}=\bigcup_{j=1}^{\infty} M^{j}$. Summing for all $k$ gives

$$
\begin{aligned}
& \sum_{j=1}^{\infty} f\left(N^{k} \cap S_{1}, N^{k} \cap S_{1}\right)=\sum_{k=1}^{\infty} \sum_{j=1}^{\infty} f\left(M^{j} \cap N^{k} \cap S_{1}, M^{j} \cap N^{k} \cap S_{2}\right) \\
& =\sum_{j=1}^{\infty} \sum_{k=1}^{\infty} f\left(N^{k} \cap\left(M^{j} \cap S_{1}\right), N^{k} \cap\left(M^{j} \cap S_{2}\right)\right)=\sum_{j=1}^{\infty} f\left(M^{j} \cap S_{1}, M^{j} \cap S_{2}\right)
\end{aligned}
$$

by Theorem 1 since $m_{e}\left(\left(M^{j} \cap S_{1}\right) \cup\left(M^{j} \cap S_{2}\right)\right) \leq m\left(M^{j}\right)<\infty$ and $\left(\left(M^{j} \cap S_{1}\right) \cup\left(M^{j} \cap S_{2}\right)\right) \subset\left(S_{1} \cup S_{2}\right) \subset\left(\bigcup_{k=1}^{\infty} N^{k}\right)$, the interchange of the order of summation being legitimate since all terms are nonnegative. This shows that $\sum_{k=1}^{\infty} f\left(N^{k} \cap S_{1}, N^{k} \cap S_{2}\right)$ is equal to the right-hand side of (3.13), so that the definition (3.13) of $f\left(S_{1}, S_{2}\right)$ is independent of which division of $\widetilde{M}$ as in (3.12) is used.

Also, the definition (3.13) of $f\left(S_{1}, S_{2}\right)$ is independent of which $\widetilde{M}$ as in (3.11) is used. If $m_{e}\left(S_{1}, S_{2}\right)=\infty$ and $\left(S_{1} \cup S_{2}\right) \subset \widetilde{N}$ for some $\widetilde{N} \in \widetilde{\mathcal{M}}$, and $\widetilde{N}=\bigcup_{k=1}^{\infty} N^{k}$ with $N^{k} \in \mathcal{M}$ and $m\left(N^{k}\right)<\infty$ for all $k$ and the $N^{k}$ are mutually disjoint, then

$$
\widetilde{M}=(\widetilde{M}-\widetilde{N}) \cup(\widetilde{M} \cap \widetilde{N})=\left(\bigcup_{j=1}^{\infty}\left(M^{j}-\widetilde{N}\right)\right) \cup\left(\bigcup_{j=1}^{\infty} \bigcup_{k=1}^{\infty}\left(M^{j} \cap N^{k}\right)\right)
$$

so that using $\widetilde{M}$ one has

$$
f\left(S_{1}, S_{2}\right)=\sum_{k=1}^{\infty} \sum_{j=1}^{\infty} f\left(M^{j} \cap N^{k} \cap S_{1}, M^{j} \cap N^{k} \cap S_{2}\right)
$$

since $\left(M^{j}-\widetilde{N}\right) \cap S_{1}=\emptyset,\left(M^{j}-\widetilde{N}\right) \cap S_{2}=\emptyset$ and $f(\emptyset, \emptyset)=0$.

Likewise, using $\widetilde{N}$ for the definition of $f\left(S_{1}, S_{2}\right)$, the same double sum expression is obtained for $f\left(S_{1}, S_{2}\right)$. This shows that the value of $f\left(S_{1}, S_{2}\right)$ is independent of which $\bar{M}$ is used in (3.11). Incidentally, note that if $\left(S_{1} \cup S_{2}\right) \subset \widetilde{M}$ and $m_{e}\left(S_{1} \cup S_{2}\right)<\infty$, then the 
formulas (3.13) and (3.12) are correct formulas, by Theorem 1 already proved when $m_{e}\left(S_{1} \cup S_{2}\right)<\infty$, since $\left(X-\bigcup_{j=1}^{\infty} M^{j}\right) \cap S_{1}=$ $\emptyset,\left(X-\bigcup_{j=1}^{\infty} M^{j}\right) \cap S_{2}=\emptyset$, and $f(\emptyset, \emptyset)=0$.

The following theorem has been proved above.

Theorem 2. Consider two disjoint sets $S_{1}$ and $S_{2}$ which are contained in $\widetilde{M}$ for some $\widetilde{M} \in \widetilde{\mathcal{M}}$, where $\widetilde{\mathcal{M}}$ is defined in (2.10) and (2.8). Then there are non-negative functionals of $S_{1}, S_{2}$, namely $d_{i}\left(S_{1}, S_{2}\right), d_{e}\left(S_{1}, S_{2}\right), g_{1}\left(S_{1}, S_{2}\right), g_{2}\left(S_{1}, S_{2}\right)$ and $h\left(S_{1}, S_{2}\right)$, i.e.,

$$
\begin{cases}f\left(S_{1}, S_{2}\right) \geq 0, & \text { where } f\left(S_{1}, S_{2}\right) \text { is any one of } \\ & d_{i}\left(S_{1}, S_{2}\right), d_{e}\left(S_{1}, S_{2}\right), g_{1}\left(S_{1}, S_{2}\right), \\ & g_{2}\left(S_{1}, S_{2}\right), h\left(S_{1}, S_{2}\right) .\end{cases}
$$

These are defined in (3.2) when $m_{e}\left(S_{1} \cup S_{2}\right)<\infty$, and in (3.12), (3.13) when $m_{e}\left(S_{1} \cup S_{2}\right)=\infty$.

Returning to Theorem 1, it has been proved above, after its statement, when $m_{e}\left(S_{1} \cup S_{2}\right)<\infty$. Consider now the case that $m_{e}\left(S_{1} \cup S_{2}\right)=\infty$. Then $f\left(S_{1}, S_{2}\right)$ is given by (3.12), (3.13). Since $m_{e}\left(\left(M^{j} \cap S_{1}\right) \cup\left(M^{j} \cap S_{2}\right)\right) \leq m\left(M^{j}\right)<\infty$, one has by (3.5) that

$$
\begin{aligned}
& f\left(M^{j} \cap S_{1}, M^{j} \cap S_{2}\right)=\sum_{k} f\left(L_{k} \cap M^{j} \cap S_{1}, L_{k} \cap M^{j} \cap S_{2}\right) \\
& \quad+f\left(\left(X-\bigcup_{k} L_{k}\right) \cap M^{j} \cap S_{1},\left(X-\bigcup_{k} L_{k}\right) \cap M^{j} \cap S_{2}\right) .
\end{aligned}
$$

Summing for all $j$ from 1 to $\infty$, one obtains

$$
\begin{gathered}
\sum_{j=1}^{\infty} f\left(M^{j} \cap S_{1}, M^{j} \cap S_{2}\right)=\sum_{k} \sum_{j=1}^{\infty} f\left(L_{k} \cap M^{j} \cap S_{1}, L_{k} \cap M^{j} \cap S_{2}\right) \\
+\sum_{j=1}^{\infty} f\left(\left(X-\bigcup_{k} L_{k}\right) \cap M^{j} \cap S_{1},\left(X-\bigcup_{k} L_{k}\right) \cap M^{j} \cap S_{2}\right),
\end{gathered}
$$


having interchanged the order of summation, since all terms are non-negative. From (3.13), this is

$$
\begin{aligned}
& f\left(S_{1}, S_{2}\right)=\sum_{k} f\left(L_{k} \cap S_{1}, L_{k} \cap S_{2}\right) \\
& +f\left(\left(X-\bigcup_{k} L_{k}\right) \cap S_{1},\left(X-\bigcup_{k} L_{k}\right) \cap S_{2}\right)
\end{aligned}
$$

which is (3.5). Theorem 1 is completely proved.

Incidentally, formulas (3.2) and (3.3) can be written in a form which is valid even if $m_{e}\left(S_{1} \cup S_{2}\right)=\infty$. Namely, transpose all the negative terms in these formulas to the other side of the equations, resulting in

$$
\left\{\begin{array}{l}
d_{i}\left(S_{1}, S_{2}\right)+m_{i}\left(S_{1}\right)+m_{i}\left(S_{2}\right)=m_{i}\left(S_{1} \cup S_{2}\right), \\
d_{e}\left(S_{1}, S_{2}\right)+m_{e}\left(S_{1} \cup S_{2}\right)=m_{e}\left(S_{1}\right)+m_{e}\left(S_{2}\right), \\
g_{1}\left(S_{1}, S_{2}\right)+m_{i}\left(S_{1}\right)+m_{e}\left(S_{2}\right)=m_{e}\left(S_{1} \cup S_{2}\right), \\
g_{2}\left(S_{1}, S_{2}\right)+m_{i}\left(S_{2}\right)+m_{e}\left(S_{1}\right)=m_{e}\left(S_{1} \cup S_{2}\right), \\
h\left(S_{1}, S_{2}\right)+m_{i}\left(S_{1} \cup S_{2}\right)+m_{e}\left(S_{1} \cup S_{2}\right) \\
=m_{i}\left(S_{1}\right)+m_{e}\left(S_{1}\right)+m_{i}\left(S_{2}\right)+m_{e}\left(S_{2}\right),
\end{array}\right.
$$

and

$$
h\left(S_{1}, S_{2}\right)+d_{i}\left(S_{1}, S_{2}\right)=d_{e}\left(S_{1}, S_{2}\right) .
$$

These are true when $m_{e}\left(S_{1} \cup S_{2}\right)<\infty$, and also when $m_{e}\left(S_{1} \cup S_{2}\right)=$ $\infty$ and $\left(S_{1} \cup S_{2}\right) \subset \widetilde{M} \in \widetilde{\mathcal{M}}$, by writing (3.15), (3.16) for the sets $M^{j} \cap S_{1}, M^{j} \cap S_{2}$ and then summing over all $j$ from 1 to $\infty$, using (3.12), (3.13) and Lemma 1 for the sums involving $m_{i}$ and $m_{e}$.

The inequality $d_{i}\left(S_{1}, S_{2}\right) \leq d_{e}\left(S_{1}, S_{2}\right)$, or $h\left(S_{1}, S_{2}\right) \geq 0$, can be stated in an interesting form. For any set $S$ define the average measure of $S$, written $m_{a}(S)$, by

$$
m_{a}(S)=\frac{1}{2}\left(m_{i}(S)+m_{e}(S)\right)
$$

it being supposed that there is an $M \in \mathcal{M}$ with $S \subset M$. Note that $\frac{1}{2} m_{e}(S) \leq m_{a}(S) \leq m_{e}(S)$. Consider two disjoint sets $S_{1}, S_{2}$ for which $m_{e}\left(S_{1}\right)<\infty, m_{a}\left(S_{2}\right)<\infty$. The statement that $h\left(S_{1}, S_{2}\right) \geq 0$ can be written in the form

$$
m_{a}\left(S_{1} \cup S_{2}\right) \leq m_{a}\left(S_{1}\right)+m_{a}\left(S_{2}\right) .
$$


This follows from the formula for $h\left(S_{1}, S_{2}\right)$ in (3.2). If $m_{e}\left(S_{j}\right)=\infty$ for $j=1$ or 2 or both, formula (3.18) is still true since then $m_{a}\left(S_{j}\right)=\infty$. Thus, $m_{a}(S)$ is subadditive; and it is also nonnegative and monotone increasing. Incidentally, the subadditivity (3.18) also holds if $S_{1}$ and $S_{2}$ are not disjoint, since

$$
\begin{aligned}
m_{a}\left(S_{1} \cup S_{2}\right)=m_{a}\left(S_{1} \cup\left(S_{2}-S_{1}\right)\right) & \leq m_{a}\left(S_{1}\right)+m_{a}\left(S_{2}-S_{1}\right) \\
& \leq m_{a}\left(S_{1}\right)+m_{a}\left(S_{2}\right) .
\end{aligned}
$$

Indeed, $m_{a}(S)$ is countably subadditive, as in the following theorem.

THEOREM 3. The average measure $m_{a}(S)$ of a set $S$, defined in (3.17), is subadditive and indeed is countably subadditive. That is, if $S_{j}$, for $j=1,2, \ldots$ to $n$ or to $\infty$, is a countable number of sets such that $\left(\bigcup_{j} S_{j}\right) \subset M$ for some $M \in \mathcal{M}$, then

$$
m_{a}\left(\bigcup_{j} S_{j}\right) \leq \sum_{j} m_{a}\left(S_{j}\right)
$$

Proof. The subadditivity of $m_{a}(S)$ for two sets has been proved above, in (3.18). The proof for any countable number $n$ or $\infty$ of sets $S_{j}$ is given in [2], in the last paragraph on page 370 and on page 371 of [2]. (There is a misprint in the middle of page 371 of [2], in formula (5.1) there. The right-hand side of (5.1) should be $\sum_{\underline{\nu}}^{\infty} m_{a}\left(S_{\underline{\nu}}\right)+\epsilon$.) The same proof holds for the general measure space $(X, \mathcal{M}, m)$, replacing the words "measurable set" by "set in $\mathcal{M}^{\prime \prime}$.

Also, the complementation and other properties of $m_{a}(S)$, as in the lower part of page 371 and the top paragraph of page 372 of [2], hold in the general measure space $(X, \mathcal{M}, m)$.

Returning to the non-negative functionals of two disjoint sets it will be shown in a later section of the present article that the four functionals $d_{i}\left(S_{1}, S_{2}\right), g_{1}\left(S_{1}, S_{2}\right), g_{2}\left(S_{1}, S_{2}\right)$, and $h\left(S_{1}, S_{2}\right)$ are independent functionals, while $d_{e}\left(S_{1}, S_{2}\right)$ is expressible in terms of them 
by (3.16). To simplify the writing, place

$$
\begin{cases}a_{1}=m_{i}\left(S_{1}\right), & a_{2}=m_{i}\left(S_{2}\right), a=m_{i}\left(S_{1} \cup S_{2}\right), \\ b_{1}=m_{e}\left(S_{1}\right), & b_{2}=m_{e}\left(S_{2}\right), b=m_{e}\left(S_{1} \cup S_{2}\right),\end{cases}
$$

and

$$
\begin{cases}d_{i}=d_{i}\left(S_{1}, S_{2}\right), & d_{e}=d_{e}\left(S_{1}, S_{2}\right), \\ g_{1}=g_{1}\left(S_{1}, S_{2}\right), & g_{2}=g_{2}\left(S_{1}, S_{2}\right), h=h\left(S_{1}, S_{2}\right),\end{cases}
$$

all of which are non-negative real numbers or $\infty$. For the case that $m_{e}\left(S_{1}, S_{2}\right)<\infty$, the four formulas in (3.2) for $d_{i}, g_{1}, g_{2}$ and $h$ (omitting the $d_{e}$ ) can be solved for the four quantities $a, b_{1}, b_{2}, b$ with the result given in (3.23) shortly below, and also $d_{e}=d_{i}+h$. This is easily done, and is done on pages 374,375 of [2], which use the same letters as above. Thus, the quantities $b_{1}, b_{2}, a, b$ can be expressed in terms of the six quantities $a_{1}, a_{2}, d_{i}, h, g_{1}, g_{2}$ by (3.23), and so can $a_{1}$ and $a_{2}$ by $a_{1}=a_{1}, a_{2}=a_{2}$. The formulas (3.22) below follow from (3.23), since by (3.23) one has

$$
a_{1}+a_{2}+d_{i}=a=m_{i}\left(S_{1} \cup S_{2}\right) \leq m_{i}(S)
$$

and

$$
a_{1}+a_{2}+d_{i}+h+g_{1}+g_{2}=b=m_{e}\left(S_{1} \cup S_{2}\right) \leq m_{e}(S) .
$$

The following theorem has been obtained above.

THEOREM 4. Suppose that $S_{1}$ and $S_{2}$ are two disjoint sets, $S_{1} \cap S_{2}=\emptyset$, in a measure space $(X, \mathcal{M}, m)$, and that

$$
\left(S_{1} \cup S_{2}\right) \subset S \text {, where } S \subset \widetilde{M} \text { for some } \widetilde{M} \subset \widetilde{\mathcal{M}} \text {, }
$$

where $\widetilde{\mathcal{M}}$ is defined in (2.8) and (2.10). Then there are 6 set functions, whose values are non-negative real numbers or $\infty$, namely

$$
\begin{array}{r}
m_{i}\left(S_{1}\right), m_{i}\left(S_{2}\right), d_{i}\left(S_{1}, S_{2}\right), \\
g_{1}\left(S_{1}, S_{2}\right), g_{2}\left(S_{1}, S_{2}\right), h\left(S_{1}, S_{2}\right),
\end{array}
$$

all $\geq 0$, which satisfy

$$
\left\{\begin{array}{l}
a_{1}+a_{2}+d_{i} \leq m_{i}(S), \\
a_{1}+a_{2}+d_{i}+g_{1}+g_{2}+h \leq m_{e}(S),
\end{array}\right. \text { and }
$$


where the letters in (3.22) have the meaning given in (3.19) and (3.20). The 6 quantities $m_{i}(S)$ and $m_{e}(S)$, for $S=S_{1}, S_{2}$, and $\left(S_{1} \cup S_{2}\right)$, can be expressed in terms of the six quantities in (3.21) by the formulas:

$$
\left\{\begin{array}{l}
b_{1}=a_{1}+d_{i}+h+g_{1}, \\
b_{2}=a_{2}+d_{i}+h+g_{2}, \\
a=a_{1}+a_{2}+d_{i} \\
b=a_{1}+a_{2}+d_{i}+h+g_{1}+g_{2}
\end{array}\right.
$$

and also $a_{1}=a_{1}, a_{2}=a_{2}$.

It will be shown in a later section of the present article that if the measure space $(X, \mathcal{M}, m)$ satisfies some conditions, which are satisfied in the usual measure spaces, then the 6 set functions $a_{1}, a_{2}, d_{i}, g_{1}, g_{2}, h$ are a complete collection of independent non-negative set functions.

Incidentally, concerning the set functions $f\left(S_{1}, S_{2}\right)$, a consequence of Theorem 1 is the following. For given disjoint sets $S_{1}, S_{2}$ with $\left(S_{1} \cup S_{2}\right) \subset \widetilde{M} \in \widetilde{\mathcal{M}}$, consider the functional $f\left(M \cap S_{1}, M \cap S_{2}\right)$ as a functional of the set $M \in \mathcal{M}$. If $M=\bigcup_{k} M_{k}$, and $M_{k} \in \mathcal{M}$ for all $k$ and the $M_{k}$ are mutually disjoint, then

$$
f\left(M \cap S_{1}, M \cap S_{2}\right)=\sum_{k} f\left(M_{k} \cap S_{1}, M_{k} \cap S_{2}\right) .
$$

For, in Theorem 1,replace $S_{1}, S_{2}$ by $M \cap S_{1}, M \cap S_{2}$ respectively, and note that $M_{k} \cap M=M_{k}$ and $\left(X-\bigcup_{k} M_{k}\right) \cap M=\emptyset$. Formula (3.24) and Theorem 1 state that the non-negative functional $f(M \cap$ $S_{1}, M \cap S_{2}$ ), when considered as a functional of the set $M \in \mathcal{M}$, is countably additive for disjoint sets $M \in \mathcal{M}$. This is like $m(M)$, and also $m_{i}(M \cap S)$ and $m_{e}(M \cap S)$ by Lemma 1 . And also note that, if $A$ and $B$ are two sets $\in \mathcal{M}$ with $A \subset B$, then

$$
f\left(A \cap S_{1}, A \cap S_{2}\right) \leq f\left(B \cap S_{1}, B \cap S_{2}\right),
$$

since

$f\left(B \cap S_{1}, B \cap S_{2}\right)=f\left(A \cap S_{1}, A \cap S_{2}\right)+f\left((B-A) \cap S_{1},(B-A) \cap S_{2}\right)$

by (3.24) for two sets $M_{k}$. 
4. Partitionable Sets. Consider a general measure space $(X, \mathcal{M}, m)$, and a set $M \in \mathcal{M}$ with $m(M)>0$. The set $M$ will be called a partitionable set if, for each set $B$ in $\mathcal{M}$ with $B \subset M$ and $m(B)>0$, there is a set $A \in \mathcal{M}$, where $A$ depends on $B$, with

$$
A \subset B \quad \text { and } \quad 0<m(A)<m(B) .
$$

Note that if $M$ is partitionable, then every set $B \subset M$ with $B \in \mathcal{M}$ and $m(B)>0$ is also partitionable. A measure space $(X, \mathcal{M}, m)$ for which every set $M \in \mathcal{M}$, with $m(M)>0$, is partitionable will be called a partitionable measure space. The following lemma will first be proved.

Lemma 3. Suppose that $M \in \mathcal{M}$ is a partitionable set with $m(M)>0$, in a measure space $(X, \mathcal{M}, m)$. Then for any positive real number $r$, there is a set

$$
A \in \mathcal{M}, A \subset M, \text { and } 0<m(A) \leq r .
$$

Proof. Consider first the case that $m(M)<\infty$. There is a set $B \in \mathcal{M}, B \subset M$ with $0<m(B)<m(M)$, since $M$ is a partitionable set. Then $m(M)=m(B)+m(M-B)$, and at least one of the sets $B$ or $M-B$ has its $m$-measure $\leq \frac{1}{2} m(M)$. If $r \geq \frac{1}{2} m(M)$, then this set can be taken for the set $A$. For any positive $r$, let $n$ be a positive integer such that $\frac{1}{2^{n}} m(M) \leq r$. There is a set $A_{1} \in \mathcal{M}, A_{2} \subset A_{1}$, with $0<m\left(A_{2}\right) \leq \frac{1}{2} m\left(A_{1}\right) \leq \frac{1}{2^{2}} m(M)$; etc., etc.; at the $n$-th step, there is a set $A_{n} \in \mathcal{M}, A_{n} \in M$, with $0<m\left(A_{n}\right) \leq \frac{1}{2^{n}} m(M) \leq r$. The set $A_{n}$ is a set $A$ of the lemma. If $m(M)=\infty$, there is a set $B \subset M, B \in \mathcal{M}$ with $0<m(B)<\infty$, by the partitionability of $M$. Pick a set $A \subset B, A \in \mathcal{M}$ with $0<m(A) \leq r$. Lemma 3 is proved.

TheOREM 5. Suppose that $M \in \mathcal{M}$ is a partitionable set, with $m(M)>0$, in a measure space $(X, \mathcal{M}, m)$. Then, if $c$ is any positive real number or $\infty$ with $c \leq m(M)$, there is a set $C \in \widetilde{\mathcal{M}}, C \subset M$, with $m(C)=c$. $(\widetilde{\mathcal{M}}$ is defined in (2.10) and (2.8)). If $m(M)=\infty$ 
and $c=\infty$, there is a set $C \in \widetilde{\mathcal{M}}, C \subset M$ with $m(C)=\infty$ and $m(M-C)=\infty$.

Proof. (If $c=0$, the set $\emptyset$ will do, and consider $c>0$.) There is by Lemma 3 , a set $C_{1} \in \widetilde{\mathcal{M}}, C_{1} \subset M$ with $0<m\left(C_{1}\right) \leq \min (c, 1)$, where $\min (c, 1)$ is the minimum of $c$ and 1 . Then $m\left(M-C_{1}\right)=$ $m(M)-m\left(C_{1}\right) \geq c-m\left(C_{1}\right) \geq 0$. If $c-m\left(C_{1}\right)=0$, pick $C_{2}=\emptyset$. If $c-m\left(C_{1}\right)>0$, there is by Lemma 2 a set $C_{2} \in \widetilde{\mathcal{M}}, C_{2} \subset\left(M-C_{1}\right)$ with $0<m\left(C_{2}\right) \leq \min \left(c-m\left(C_{1}\right), 1\right)$; then

$$
\begin{aligned}
m\left(M-\left(C_{1} \cup C_{2}\right)\right) & =m\left(\left(M-C_{1}\right)-C_{2}\right) \\
& =m\left(M-C_{1}\right)-m\left(C_{2}\right) \\
& \geq c-m\left(C_{1}\right)-m\left(C_{2}\right) \geq 0 .
\end{aligned}
$$

If $c-m\left(C_{1}\right)-m\left(C_{2}\right)=0$, pick $C_{3}=\emptyset$; if $c-m(C-1)-m\left(C_{2}\right)>0$, pick $C_{3} \in \widetilde{\mathcal{M}}, C_{3} \subset\left(M-\left(C_{1} \cup C_{2}\right)\right)$ with $0<m\left(C_{3}\right) \leq \min (c-$ $\left.m\left(C_{1}\right)-m\left(C_{2}\right), 1\right)$. Continue by transfinite induction over countable ordinal numbers. For any countable ordinal number $\underline{\gamma}$, suppose that sets $C_{\underline{\alpha}} \in \widetilde{\mathcal{M}}, C_{\underline{\alpha}} \subset M$, have been selected for all ordinals $\underline{\alpha}<\underline{\gamma}$, such that for every ordinal $\underline{\alpha}<\underline{\gamma}$ :

$$
\left\{\begin{array}{l}
C_{\underline{\alpha}} \subset\left(M-\bigcup_{\underline{\beta}<\underline{\alpha}} C_{\underline{\beta}}\right), \\
\sum_{\underline{\beta}<\underline{\alpha}} m\left(C_{\underline{\beta}}\right) \leq c, \\
\text { and if } \sum_{\underline{\beta}<\underline{\alpha}} m\left(C_{\underline{\beta}}\right)=c \quad \text { then } C_{\underline{\alpha}}=\emptyset \\
\text { while if } \sum_{\underline{\beta}<\underline{\alpha}} m\left(C_{\underline{\beta}}\right)<c \quad \text { then } 0<m\left(C_{\underline{\alpha}}\right) \\
\quad \leq \min \left(c-\sum_{\underline{\beta}<\underline{\alpha}} m\left(C_{\underline{\beta}}\right), 1\right) .
\end{array}\right.
$$

The sets $C_{1}, C_{2}, C_{3}$ have been obtained above, and satisfy (4.3) for $\underline{\gamma}=1,2,3$. If $\sum_{\underline{\alpha}<\underline{\gamma}} m\left(C_{\underline{\alpha}}\right)=c$, select $C_{\underline{\gamma}}=\emptyset$. If $\sum_{\underline{\alpha}<\underline{\gamma}} m\left(C_{\underline{\alpha}}\right)<c$, then $\left(\bigcup_{\alpha<\underline{\alpha}} C_{\underline{\alpha}}\right) \in \widetilde{\mathcal{M}}$ since $\underline{\gamma}$ is a countable ordinal number, and $m\left(M-\bigcup_{\underline{\alpha}<\underline{\underline{\gamma}}}^{\underline{\alpha}<\underline{\gamma}} C_{\underline{\alpha}}\right)=m(M)-m\left(\bigcup_{\underline{\alpha}<\underline{\underline{\gamma}}} C_{\underline{\alpha}}\right) \geq c-m\left(\bigcup_{\underline{\alpha}<\underline{\underline{\gamma}}} C_{\underline{\alpha}}\right)>0$, and 
select $C_{\underline{\gamma}} \in \widetilde{\mathcal{M}}, C_{\underline{\gamma}} \subset\left(M-\bigcup_{\underline{\alpha}<\underline{\underline{\alpha}}} C_{\underline{\alpha}}\right)$ with $0<m\left(C_{\underline{\gamma}}\right) \leq \min (c-$ $\left.m\left(\bigcup_{\underline{\alpha}<\underline{\gamma}} C_{\underline{\alpha}}\right), 1\right)$. Then (4.3) holds for $\alpha=\gamma$ also.

By the principle of transfinite induction, the mutually disjoint sets $C_{\underline{\alpha}} \in \widetilde{\mathcal{M}}$ and $C_{\underline{\alpha}} \subset M$ have been selected for all countable ordinal numbers, with (4.3) being satisfied for all these ordinals $\underline{\alpha}$. And for every countable ordinal $\underline{\alpha}$, either $C_{\underline{\alpha}}=\emptyset$ or $0<m\left(C_{\underline{\alpha}}\right) \leq 1$.: For any given positive integer $n$, there are only a countable number of $C_{\underline{\alpha}}$ with $m\left(C_{\underline{\alpha}} \geq \frac{1}{2^{n}}\right)$. For, if there are an infinity of them, as $C_{\underline{\alpha}_{1}}, C_{\underline{\alpha}_{2}}, \ldots, C_{\underline{\alpha}_{1}}, \ldots$ where $\underline{\alpha}_{1}<\underline{\alpha}_{2}<\ldots<\underline{\alpha}_{j}<\underline{\alpha}_{j+1}<\ldots$ for all integral $j$ from 1 to $\infty$. Then $m\left(\bigcup_{j=1}^{\infty} C_{\underline{\alpha}_{\jmath}}\right)=\sum_{j=1}^{\infty} m\left(C_{\underline{\alpha}_{\jmath}}\right)=\infty$ since $m\left(C_{\underline{\alpha}_{j}}\right) \geq \frac{1}{2^{n}}$ for every $j$. If $\underline{\gamma}$ is any countable ordinal $>\underline{\alpha}_{j}$ for all j, i.e. $\underline{\gamma} \geq \lim _{j \rightarrow \infty} \underline{\alpha}_{j}$, then $m\left(\bigcup_{\underline{\alpha}<\underline{\gamma}} C_{\underline{\alpha}}\right) \geq m\left(\bigcup_{j=1}^{\infty} C_{\underline{\alpha}}\right)=\infty$, and $c=\infty$, so that $C_{\gamma}=\emptyset$ by (4.3). In any case, there are at most a countable infinity of $C_{\underline{\alpha}}$ for which $m\left(C_{\underline{\alpha}}\right)>0$. Since the number of countable ordinals is uncountably infinite, there are countable ordinals larger than all these. Let $\gamma$ be the smallest countable ordinal such that $m\left(C_{\underline{\gamma}}\right)=0$, so that $\sum_{\underline{\beta}<\underline{\gamma}} m\left(C_{\underline{\beta}}\right)=c$ by $(4.3)$.

Place $C=\bigcup_{\underline{\beta}<\underline{\gamma}} C_{\underline{\beta}}$, which $\in \widetilde{\mathcal{M}}$ by $(2.8)$ and (2.10) since $\underline{\gamma}$ is countable ordinal number. Theorem 5, up to the last sentence, is proved. For the last sentence of Theorem 5, which is the case $m(M)=\infty$ and $c=\infty$, one has $C \in \widetilde{\mathcal{M}}, C \subset M$, with $m(C)=$ $\infty=m(M)$. Now, by $(2.9), C=\bigcup_{j=1}^{\infty} M^{j}$, where the $M^{j}$ are mutually disjoint and $m\left(M^{j}\right)<\infty$ for all $j$. By the first part of Theorem 5 already proved, there is $A^{j} \in M, A^{j} \subset M^{j}$ with $m\left(A^{j}\right)=\frac{1}{2} m\left(M_{\infty}^{j}\right)$, and therefore $m\left(M^{j}-A^{j}\right)=\frac{1}{2} m\left(M^{j}\right)$. Then, the set $A=\bigcup_{j=1}^{\infty} A^{j} \in$ $\widetilde{\mathcal{M}}$ and $A \subset C \subset M$ and has $m(A)=\sum_{j=1}^{\infty} m\left(A^{j}\right)=\frac{1}{2} \sum_{j=1}^{\infty} m\left(M^{j}\right)=$ 
$\frac{1}{2} m(C)=\infty$. Also, $(C-A)=\bigcup_{j=1}^{\infty}\left(M^{j}-A^{j}\right)$ and $m(C-A)=$ $\sum_{j=1}^{\infty} m\left(M^{j}-A^{j}\right)=\sum_{j=1}^{\infty} \frac{1}{2} m\left(M^{j}\right)=\infty$. Theorem 5 is proved, with the set $A$ as the $C$ of the last sentence of the theorem.

One easily shows from Theorem 5 that, for a partitionable set $M \in \mathcal{M}$ with $m(M)>0$, if $c_{j} \geq 0, j=1,2, \ldots$ to $n$ or to $\infty$, are a countable number of non-negative real numbers or $\infty$ such that $\sum_{j} c_{j} \leq m(M)$, then there are mutually disjoint sets $C_{j} \in \widetilde{\mathcal{M}}$ with $C_{j} \subset M$ and $m\left(C_{j}\right)=c_{j}$, for all $j=1,2, \ldots$ to $n$ or to $\infty$. A proof is given on page 384 of the Pacific Journal of Mahtematics article [2], Lemma 8.

There is also the following lemma.

Lemma 4. A partitionable set $M \in \widetilde{\mathcal{M}}$ with $m(M)>0$ contains an uncountable infinity of points. If the partitionable set $M \in \widetilde{\mathcal{M}}$, then any point $x$ in $M$ is contained in a set $M_{0} \in \mathcal{M}, M_{0} \subset M$, with $m\left(M_{0}\right)=0$.

Proof. Considering the second sentence of Lemma 4, suppose first that $0<m(M)<\infty$. (If $m(M)=0$, then $M_{0}$ can be taken as $M$.) There is by Theorem 5 a set $C \in \widetilde{\mathcal{M}}$ with $C \subset M$ and $m(C)=\frac{1}{2} m(M)$, and then $m(M-C)=\frac{1}{2} m(M)$ also. One of the sets $C$ or $M-C$ contains the point $x$, and designating this one by $A_{1}$ one has $A_{1} \in \widetilde{\mathcal{M}}, A_{1} \subset M, x \in A_{1}$, and $m\left(A_{1}\right)=\frac{1}{2} m(M)$. Then there is $A_{2} \in \widetilde{\mathcal{M}}, A_{2} \subset A_{1}, x \in A_{2}$, and $m\left(A_{2}\right)=\frac{1}{2} m\left(A_{1}\right)=$ $\frac{1}{2^{2}} m(M)$; and there is $A_{3} \in \widetilde{\mathcal{M}}, A_{3} \subset A_{2}, x \in A_{3}$, and $m\left(A_{3}\right)=$ $\frac{1}{2} m\left(A_{2}\right)=\frac{1}{2^{3}} m(M)$. Continuing for all possible integers $n$, there are $A_{n} \in \widetilde{\mathcal{M}}, A_{n} \subset A_{n-1}, x \in A_{n}$, and $m\left(A_{n}\right)=\frac{1}{2^{n}} m(M)$. Placing $M_{0}=\left(\bigcap_{n=1}^{\infty} A_{n}\right) \subset M$, then $M_{0} \in \mathcal{M}, x \in M_{0}$, and $m\left(M_{0}\right)=0$, which is a desired $M_{0}$. If $m(M)=\infty$, then since $M \in \widetilde{\mathcal{M}}$ one has $M=\bigcup_{j=1}^{\infty} M^{j}$ by $(2.9)$, where the $M^{j}$ are mutually disjoint sets 
$\in \mathcal{M}$ with $0<m\left(M^{j}\right)<\infty$, so that $x \in M^{j_{1}}$ for some $j_{1}$. By the preceding there is $M_{0} \in \mathcal{M}$ with $M_{0} \subset M^{j !} \subset M, x \in M_{0}$, and $m\left(M_{0}\right)=0$. The second sentence of Lemma 4 is proved.

For the first sentence of Lemma 4, there is a set $B \in \mathcal{M}, B \subset M$ with $0<m(B)<m(M)$. The set $B \in \widetilde{\mathcal{M}}$ since $m(B)<\infty$, and each point $x$ in $B$ is contained in a set $M_{0} \in \mathcal{M}, M_{0} \subset B$, with $x \in M_{0}$ and $m\left(M_{0}\right)=0$. If there were only a countable number of points $x$ in $B$, then $B$ would be a union of a countable number of sets $M_{0}$ with $m\left(M_{0}\right)=0$, and one would have $m(B)=0$ contrary to $m(B)>0$. Thus $B$, and therefore $M$, contains an uncountable infinity of points. Lemma 4 is proved.

Lemma 4 is stated since one could have a two-valued measure space $(X, \mathcal{M}, m)$ which doesn't contain an infinity of points. Twovalued measure spaces are discussed in $\S 6$.

5. A Basis Condition for a Measure Space. Consider a general measure space $(X, \mathcal{M}, m)$. A measure basis of $(X, \mathcal{M}, m)$ will be defined as a collection $\mathcal{C}$ of sets $C_{\underline{\alpha}}$ in $\mathcal{M}$ with $m\left(C_{\underline{\alpha}}\right)>0$, such that every set $M$ in $\mathcal{M}$ with $m(M)>0$ contains a set $C_{\underline{\alpha}}$ in $\mathcal{C}$, $C_{\underline{\alpha}} \subset M$ (where $C_{\underline{\alpha}}$ depends on $M$ ). There is at least one such collection $\mathcal{C}$, namely all sets $M$ in $\mathcal{M}$ with $m(M)>0$. Consider the cardinal number of all the different sets $C_{\underline{\alpha}}$ in such collection $\mathcal{C}$, and let $\chi^{(f)}$ be the least cardinal number for all such collections $C$. Or, it suffices to let $\chi^{(f)}$ be the cardinal number of one such collection $\mathcal{C}$. Let $w^{(f)}$ be the least ordinal number with the cardinality $\chi^{(f)}$. There is a collection $\mathcal{C}^{(f)}$ of cardinality $\chi^{(f)}$, called a basis collection, and let $C_{\underline{\alpha}}$ denote all the sets $C_{\underline{\alpha}}$ with $m\left(C_{\underline{\alpha}}\right)>0$ in this collection $\mathcal{C}^{(f)}$, where $\underline{\alpha}$ runs over all the non-negative ordinal numbers $\underline{\alpha}<w^{(f)}$.

Consider the cardinal number of points in a set $M \in \mathcal{M}$ with $m(M)>0$, and let $\chi^{(p)}$ denote the least cardinal number of points in such sets $M$, among all sets $M \in \mathcal{M}$ with $m(M)>0$. Thus, every set $M \in \mathcal{M}$ with $m(M)>0$ has at least $\chi^{(p)}$ points.

The measure space $(X, \mathcal{M}, m)$ will be said to satisfy the basis condition if

$$
\chi^{(p)} \geq \chi^{(f)}
$$


i.e. if the number of points in every set $M \in \mathcal{M}$ with $m(M)>0$ is at least $\chi^{(f)}$.

For the usual measure spaces $(X, \mathcal{M}, m)$, the cardinal numbers $\chi^{(p)}$ and $\chi^{(f)}$ are both $2^{\chi_{0}}$, the cardinal number of the continuum, so that the usual measure spaces do satisfy the basis condition. For, consider Borel or Lebesgue measure on the real number line or in Euclidean space. There is a measure basis consisting of all bounded closed sets of positive measure. Here $\chi^{(f)}=2^{\chi_{0}}$ (since closed sets are complements of open sets, and the number of these is $\left.2^{\chi_{0}}\right)$. And every measurable set $M$ of positive measure contains a bounded closed set of positive measure, and thus contains a continuum number $2^{\chi_{0}}$ of points. So, $\chi^{(f)}=\chi^{(p)}=2^{\chi_{0}}$, and the basis condition (5.1) is satisfied.

Consider a general measure space $(X, \mathcal{M}, m)$ and suppose that:

$$
\left\{\begin{array}{l}
\text { there is at least one set } M \in \mathcal{M} \\
\text { with } m(M)>0 ; \text { and every set } M \in \mathcal{M} \\
\text { with } m(M)>0 \text { has an infinity of points. }
\end{array}\right.
$$

This is stated since one could have a two-valued measure space with a finite number of points. Two-valued measure spaces are discussed in $\S 6$. The following theorem will be proved.

THEOREM 6. Suppose that $(X, \mathcal{M}, m)$ is a measure space satisfying the basis condition (5.1), and also (5.2). Then there are $\chi^{(p)}$ mutually disjoint sets $Z_{\beta} \subset X$, where $\underline{\beta}$ varies over all ordinal numbers of cardinality $<\chi^{(p)}$, such that

$$
X=\bigcup_{\underline{\beta}} Z_{\underline{\beta}} \text {, where } Z_{\underline{\beta}_{1}} \cap Z_{\underline{\beta}_{2}}=\emptyset \text { for all } \underline{\beta}_{1} \neq \underline{\beta}_{2},
$$

and

$$
m_{i}\left(Z_{\underline{\beta}}\right)=0, \quad m_{i}\left(X-Z_{\underline{\beta}}\right)=0 \text { for all } \underline{\beta} .
$$

Proof. Let $\underline{w}^{(p)}$ be the smallest ordinal number of cardinality $\chi^{(p)}$ and $\underline{w}^{(p)}$ the smallest ordinal number of cardinality $\chi^{(f)}$, where $\underline{w}^{(f)} \leq \underline{w}^{(p)}$ by $(5.1)$. Since $\chi^{(f)} \chi^{(p)}=\chi^{(p)}$, the set of all ordered 
pairs $(\underline{\alpha}, \beta)$, for all non-negative ordinal numbers $\underline{\alpha}$ and $\underline{\beta}$ for which $\underline{\alpha}<\underline{w}^{(f)}$ and $\underline{\beta}<\underline{w}^{(p)}$, has the cardinality $\chi^{(p)}$ and so can be put into one-to-one correspondence with the set of all non-negative ordinal numbers $\underline{\gamma}<\underline{w}^{(p)}$. For any given $\underline{\gamma}<\underline{w}^{(p)}$, designate the $(\underline{\alpha}, \underline{\beta})$ of the associated pair $(\underline{\alpha}, \underline{\beta})$ in this one-to-one correspondence by $\underline{\alpha}(\underline{\gamma}), \underline{\beta}(\underline{\gamma})$. Thus, for any $\underline{\alpha}<\underline{w}^{(f)}$ and any $\underline{\beta}<\underline{w}^{(p)}$, there is one and only one $\underline{\gamma}<\underline{w}^{(p)}$ for which $\underline{\alpha}(\underline{\gamma})=\underline{\alpha}, \underline{\beta}\left(\underline{\gamma}_{1}\right)=\underline{\beta}$; and if $\underline{\gamma}_{1} \neq \underline{\gamma}_{2}$, then either $\underline{\alpha}\left(\underline{\gamma}_{1}\right) \neq \underline{\alpha}\left(\underline{\gamma}_{2}\right)$ or $\underline{\beta}\left(\underline{\gamma}_{1}\right) \not \underline{\beta}\left(\underline{\gamma}_{2}\right)$ or both.

Let $\mathcal{C}^{(f)}$, consisting of sets $C_{\underline{\alpha}} \in \mathcal{M}$ with $m\left(C_{\underline{\alpha}}\right)>0$ where $\underline{\alpha}$ varies over all ordinal numbers $\underline{\alpha}<\underline{w}^{(f)}$, be a measure basis of the measure space $(X, \mathcal{M}, m)$. Continuing with the proof of Theorem 6 , the following lemma will first be established.

LEMma 5. There are points $y_{\underline{\alpha}}$ and $x_{(\underline{\alpha}, \underline{\beta})}$ in $X$, for all ordinal numbers $\underline{\alpha}<\underline{w}^{(f)}$ and all ordinal numbers $\underline{\beta}<\underline{w}^{(p)}$, which are all different points, and

(5.5) $y_{\underline{\alpha}} \in C_{\underline{\alpha}}, x_{(\underline{\alpha}, \underline{\beta})} \in C_{\underline{\alpha}}$ for all $\underline{\alpha}<\underline{w}^{(f)}$ and all $\underline{\beta}<\underline{w}^{(p)}$.

Proof. For $\underline{\gamma}=0$, pick two different points $y_{\underline{\alpha}(0)}$ and $x_{(\underline{\alpha}(0), \underline{\beta}(0))}$ in $C_{\underline{\alpha}(0)}$. For $\underline{\gamma}=1$, pick two different points $y_{\underline{\alpha}(1)}$ and $x_{(\underline{\alpha}(1), \underline{\beta}(1))}$ in $C_{\underline{\alpha}(1)}-\left\{y_{\underline{\alpha}(0)}, x_{(\underline{\alpha}(0), \underline{\beta}(0))}\right\}$. Continue by transfinite induction. Suppose, for an ordinal number $\underline{\gamma}<\underline{w}^{(p)}$, that $y_{\underline{\alpha}(\underline{\delta})}$ and $x_{(\underline{\alpha}(\underline{\delta}), \underline{\beta}(\underline{\delta}))}$ have been selected for all ordinal numbers $\underline{\delta}<\underline{\gamma}$ in such a way that they are all different points, and

$$
y_{\underline{\alpha}(\underline{\delta})} \in C_{\underline{\alpha}(\underline{\delta})}, x_{(\underline{\alpha}(\underline{\delta}), \underline{\beta}(\underline{\delta}))} \in C_{\underline{\alpha}(\underline{\delta})} \text { for all } \underline{\delta}<\underline{\gamma} \text {. }
$$

Since $\underline{\gamma}<\underline{w}^{(p)}$, the total number of points $y_{\underline{\alpha}(\underline{\delta})}$ and $x_{(\underline{\alpha}(\underline{\delta}), \underline{\beta}(\underline{\delta}))}$ for all $\underline{\delta}<\underline{\gamma}$ has cardinality $<\chi^{(p)}$, and so the set

$$
C_{\underline{\alpha}(\underline{\gamma})}-\bigcup_{\underline{\delta}<\underline{\gamma}}\left\{y_{\underline{\alpha}(\underline{\delta})}, x_{(\underline{\alpha}(\underline{\delta}), \underline{\beta}(\underline{\delta}))}\right\}
$$


has cardinality $\geq \chi^{(p)}$ and is therefore an infinite set. Select two different points $y_{\underline{\alpha}(\underline{\gamma})}$ and $x_{(\underline{\alpha}(\underline{\gamma}), \underline{\beta}(\underline{\gamma}))}$ in this set:

$$
\begin{aligned}
& y_{\underline{\alpha}(\underline{\gamma})} \text { and } x_{(\underline{\alpha}(\underline{\gamma}), \underline{\beta}(\underline{\gamma}))} \text { both } \\
& \quad \in\left(C_{\underline{\alpha}(\underline{\delta})}-\bigcup_{\underline{\delta}<\underline{\gamma}}\left\{y_{\underline{\alpha}(\underline{\delta})}, x_{(\underline{\alpha}(\underline{\delta}), \underline{\beta}(\underline{\delta}))}\right\}\right),
\end{aligned}
$$

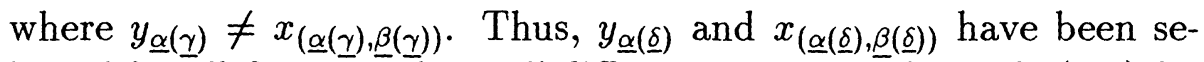
lected for all $\underline{\delta} \leq \underline{\gamma}$, and are all different points, and satisfy (5.6) for all $\underline{\delta} \leq \underline{\gamma}$. This can be done for all $\underline{\gamma}<\underline{w}^{(p)}$, and so by the principle of transfinite induction, one has selected $y_{\underline{\alpha}(\underline{\gamma})}$ and $x_{(\underline{\alpha}(\underline{\gamma}), \underline{\beta}(\underline{\gamma}))}$ for all $\underline{\gamma}<\underline{w}^{(p)}$ in such a way that they are all different points in $X$, and

$$
y_{\underline{\alpha}(\underline{\gamma})} \in C_{\underline{\alpha}(\underline{\gamma})} ; x_{(\underline{\alpha}(\underline{\gamma}), \underline{\beta}(\underline{\gamma}))} \in C_{\underline{\alpha}(\underline{\gamma})} \text { for all } \underline{\gamma}<\underline{w}^{(p)} \text {. }
$$

For any $\underline{\alpha}<\underline{w}^{(f)}$ and $\underline{\beta}<\underline{w}^{(p)}$, there is a single $\underline{\gamma}<\underline{w}^{(p)}$ such that $\underline{\alpha}(\underline{\gamma})=\underline{\alpha}$ and $\underline{\beta}(\underline{\gamma})=\underline{\beta}$, and (5.8) is (5.5). Lemma 5 is proved.

Returning to the proof of Theorem 6 , define the set $Z_{\underline{\beta}}$ by

$$
\left\{\begin{array}{l}
Z_{\underline{\beta}}=\left\{x_{(\underline{\alpha}, \underline{\beta})} ; \text { for all } \underline{\alpha}<\underline{w}^{(f)}\right\}, \quad \text { for } 0<\underline{\beta}<\underline{w}^{(p)} \\
Z_{0}=X-\bigcup_{1 \leq \underline{\beta}^{\prime} \underline{w}^{(p)}} Z_{\underline{\beta}} .
\end{array}\right.
$$

Then (5.3) is satisfied, and note that $Z_{0} \supset\left\{y_{\underline{\alpha}} ;\right.$ for all $\left.\underline{\alpha}<\underline{w}^{(f)}\right\}$. Let $M$ be a set $\in \mathcal{M}$ with $M \subset Z_{\beta}$. If $m(M)>0$, then there is a $C_{\underline{\alpha}} \in$ $\mathcal{C}^{(f)}$ with $M \supset C_{\underline{\alpha}}$, so that $C_{\underline{\alpha}} \subset Z_{\beta}$. But for $\underline{\beta}>0, y_{\underline{\alpha}} \in C_{\underline{\alpha}}$ and $y_{\underline{\alpha}} \neq Z_{\underline{\beta}}$, so that $C_{\underline{\alpha}} \not \subset Z_{\underline{\beta}}$; and for $\underline{\beta} \equiv 0, x_{(\underline{\alpha}, 1)} \in C_{\underline{\alpha}}$ and $x_{(\underline{\alpha}, 1)} \notin Z_{0}$, so that $C_{\underline{\alpha}} \not \subset Z_{0}$. These contradictions show that $m(M)=0$, and therefore $m_{i}\left(Z_{\beta}\right)=0$. Concerning the complementary set $X-Z_{\underline{\beta}}$, let $M$ be a set $\in \mathcal{M}$ with $M \subset\left(X-Z_{\underline{\beta}}\right)$. If $m(M)>0$, there is a set $C_{\underline{\alpha}} \in \mathcal{C}^{(f)}$ with $M \supset C_{\underline{\alpha}}$, so that $C_{\underline{\alpha}} \subset\left(X-Z_{\beta}\right)$. But for $\underline{\beta}>0, x_{(\underline{\alpha}, \underline{\beta})} \in C_{\underline{\alpha}}$ and $x_{(\underline{\alpha}, \underline{\beta})} \notin\left(X-Z_{\underline{\beta}}\right)$ so that $C_{\underline{\alpha}} \not \subset\left(X-Z_{\underline{\beta}}\right)$; and for $\underline{\beta}=0, \bar{y}_{\underline{\alpha}} \in C_{\underline{\alpha}}$ and $y_{\underline{\alpha}} \notin\left(X-Z_{0}\right)$ so that $C_{\underline{\alpha}} \not \subset\left(X-Z_{0}\right)$. These contradictions show that $m(M)=0$, and therefore $m_{i}\left(X-Z_{\beta}\right)=$ 0 . Thus, (5.4) holds, and Theorem 6 is proved. (Incidentally, the different points $y_{(\underline{\alpha}}$ and $x_{(\underline{\alpha}, \underline{\beta})}$ in the above proof can be replaced by mutually disjoint point sets of less than $\chi^{(p)}$ points each.) 
For Lebesgue or Borel measure on the real number line or in Euclidean space, in which case $\chi^{(p)}=\chi^{(f)}=2^{\chi_{0}}$, Theorem 6 shows that there are a continuum number of mutually disjoint sets $Z_{\beta}$ as in Theorem 6 . This is an addition to a similar theorem in the author's articles [1], [2], and in particular an addition to Theorem 7 on page 381 of [2]. Likewise for Theorem 7 below.

An immediate consequence of Theorem 6 is Theorem 7.

THEOREM 7. Suppose that $(X, \mathcal{M}, m)$ is a measure space satisfying the basis condition (5.1), and also (5.2). Then, for any integer $N \geq 2$, and also for any $\chi \leq \chi^{(p)}$, there are $N$ and also $\chi$, mutually disjoint sets $Z_{\gamma}$ for $\underline{\gamma}=0,1, \ldots, N-1$, or for $\underline{\gamma}=$ all ordinal numbers of cardinality $<\chi$, such that $X=\bigcup_{\gamma} Z_{\underline{\gamma}}, m_{i}\left(Z_{\underline{\gamma}}\right)=0$ and $m_{i}\left(X-Z_{\underline{\gamma}}\right)=0$ for all $\underline{\gamma}$. For any $M \in \mathcal{M}$ with $m(M)>0$, one has $M=\bigcup_{\underline{\gamma}}\left(M \cap Z_{\underline{\gamma}}\right)$ and

$$
\begin{aligned}
& m_{i}\left(M \cap Z_{\underline{\gamma}}\right)=0 \quad \text { and } \\
& m_{i}\left(M-\left(M \cap Z_{\underline{\gamma}}\right)\right)=0 \text { for all } \underline{\gamma}
\end{aligned}
$$

and

$$
\begin{aligned}
m_{e}\left(M \cap Z_{\underline{\gamma}}\right) & =m(M), \\
m_{e}\left(M-\left(M \cap Z_{\underline{\gamma}}\right)\right) & =m(M) \text { for all } \underline{\gamma} .
\end{aligned}
$$

Proof. The first two sentences of Theorem 7 for the case that $\chi=\chi^{(p)}$ is Theorem 6 . For the case that $\chi<\chi^{(p)}$, replace $Z_{0}$ of Theorem 6 by $\widehat{Z}_{0}=Z_{0} \cup\left(\bigcup_{\beta \geq N \text { or } \underline{w}} Z_{\underline{\beta}}\right)$, where $\underline{w}$ is the smallest ordinal number of cardinality $\chi$. Now, $\widehat{Z}_{0} \cap Z_{1}=\emptyset$ so that $\widehat{Z}_{0} \subset(X-$ $\left.Z_{1}\right)$ and $m_{i}\left(Z_{0}\right)=0$ since $m_{i}\left(X-Z_{1}\right)=0 ;$ and $\left(X-\widehat{Z}_{0}\right) \subset\left(X-Z_{0}\right)$ so that $m_{i}\left(X-\widehat{Z}_{0}\right)=0$ since $m_{i}\left(X-Z_{1}\right)=0$. This establishes the first two sentences of Theorem 7 .

Equation (5.10) is a consequence of $\left(M \cap Z_{\underline{\gamma}}\right) \subset Z_{\underline{\gamma}}$ and $(M-$ $\left.\left(M \cap Z_{\underline{\gamma}}\right)\right) \subset\left(X-Z_{\underline{\gamma}}\right)$. Also, by Lemma 2 ,

$$
m_{e}\left(M \cap Z_{\underline{\gamma}}\right)+m_{i}\left(M-\left(M \cap Z_{\underline{\gamma}}\right)\right)=m(M),
$$


and

$$
m_{i}\left(M \cap Z_{\underline{\gamma}}\right)+m_{e}\left(M-\left(M \cap Z_{\underline{\gamma}}\right)\right)=m(M),
$$

so that (5.11) follows from (5.10). Theorem 7 is proved.

(Incidentally, $\S 5$ also holds if $\chi^{(p)}$ is a finite number, which would require that the measure space be a direct sum of a finite number of 2-valued measure spaces and of a zero-measure space; and also if the second line of (5.2) is not satisfied, so that $\chi^{(p)}$ can be a finite number $\geq 2$, as can be shown. Two-valued measure spaces are discussed briefly in the latter part of $§ 6$.)

6. A Complete Set of Independent Inequalities. The following main theorem will now be proved.

THEOREM 8. Suppose that $(X, \mathcal{M}, m)$ is a partitionable measure space satisfying the basis condition (5.1). For any set $\widetilde{M}$ in $\widetilde{\mathcal{M}}$ with $m(\widetilde{M})>0$, where $\widetilde{\mathcal{M}}$ is defined in (2.10) and (2.8), and any six nonnegative real numbers or $\infty$, namely $a_{1}, a_{2}, d_{i}, h, g_{1}, g_{2}$, satisfying

$$
a_{1}+a_{2}+d_{i}+h+g_{1}+g_{2} \leq m(\widetilde{M}),
$$

there are two disjoint sets $S_{1}, S_{2}$ in $\widetilde{M}, S_{1} \subset \widetilde{M}, S_{2} \subset \widetilde{M}, S_{1} \cap S_{2}=$ $\emptyset$, such that (3.20) holds and

$$
\begin{cases}m_{i}\left(S_{1}\right)=a_{1} & \\ m_{e}\left(S_{1}\right)=a_{1}+d_{i}+h+g_{1} & \left(\text { which }=b_{1}\right), \\ m_{i}\left(S_{2}\right)=a_{2} & \\ m_{e}\left(S_{2}\right)=a_{2}+d_{i}+h+g_{2} & \left(\text { which }=b_{2}\right), \\ m_{i}\left(S_{1} \cup S_{2}\right)=a_{1}+a_{2}+d_{i} & (\text { which }=a), \\ m_{e}\left(S_{1} \cup S_{2}\right) & \\ =a_{1}+a_{2}+d_{i}+h+g_{1}+g_{2} & (\text { which }=b) .\end{cases}
$$

Proof. (A partitionable measure space is defined in the first paragraph of $\S 4$.) The theorem and proof are exactly the same as in [2]. The theorem and proof appears on pages 382-385 of that article, with the symbol $X$ in the article replaced by $\widetilde{M}$ for Theorem 8 
above, and the words "measurable set" replaced by "set in $\widetilde{\mathcal{M}}$ ". Lemma 7 in that proof appears in the present article as Theorem 5, and Lemma 1 is the same as Lemma 1 in the present article; and the sets $Z_{1}$ and $Z_{2}$ in that proof are the sets $Z_{1}$ and $Z_{2}$ in Theorem 7 for $N=3$, since $Z_{1} \cup Z_{2}=X-Z_{0}$ so that $m_{i}\left(Z_{1} \cup Z_{2}\right)=0$ and $m_{i}\left(X-\left(Z_{1} \cup Z_{2}\right)\right)=0$. A misprint appears in the cited article [2] on page 385 , line 3 ; it should read: $Z_{1} \cap Z_{2}=\emptyset$, not $Z_{1} \cap Z_{2} \neq \emptyset$. Theorem 8 is proved.

A proof of Theorem 8 is also contained in the proof of the more general Theorem 10 in $\S 7$ of the present article. Theorem 10 concerns two disjoint sets contained in any set $S$, where $S \subset \widetilde{M}$ for some $\widetilde{M}$ in $\widetilde{M}$. Theorem 8 is the case of Theorem 10 when $S=\widetilde{M}$. Then $m_{i}(S)=m_{e}(S)=m(\widetilde{M})$, and the second line of (7.11) is a consequence of the first line of (7.11), and the sets $K$ and $L$ appearing in the proof of Theorem 10 are also $=\widetilde{M}$.

Theorems 2 and 8 are the main theorems concerning the quantities $m_{i}(S)$ and $m_{e}(S)$ for $S=S_{1}, S_{2}$, and $S_{1} \cup S_{2}$, where $S_{1}$ and $S_{2}$ are disjoint sets contained in a set $\widetilde{M}$ in $\widetilde{\mathcal{M}}$ with $m(\widetilde{M})>0$. They state that the six quantities $a_{1}, b_{1}, a_{2}, b_{2}, a, b$ defined in (3.19) are subject to six independent inequalities, that $a_{1}, a_{2}, d_{i}, h, g_{1}, g_{2}$ are each $\geq 0$, and the inequality $a_{1}+a_{2}+d_{i}+h+g_{1}+g_{2} \leq m(M)$, where $b_{1}, b_{2}, a, b$ and $a_{1}, a_{2}$ are expressible in terms of them by (6.1). These are valid for every pair of disjoint sets $S_{1}, S_{2}$ contained in $\widetilde{M}$, and any other numerical relation involving $a_{1}, b_{1}, a_{2}, b_{2}, a, b$ and $m(\widetilde{M})$ which is valid for every pair of disjoint sets $\subset \widetilde{M}$ is a consequence of these. For, Theorem 8 states that the six non-negative quantities $a_{1}, a_{2}, d_{i}, h, g_{1}, g_{2}$ can have any values independently subject merely to their sum being $\leq m(\widetilde{M})$. In any particular case or cases of two disjoint sets $S_{1}, S_{2}$ in a set $M \in \widetilde{\mathcal{M}}$, there may be more information applicable to the particular case or cases.

Without the use of the symbols $d_{i}, h, g_{1}, g_{2}$, the inequalities are

$$
\left\{\begin{array}{l}
a_{1} \geq 0, \quad a_{2} \geq 0, \quad a \geq a_{1}+a_{2}, \\
a+b \geq a_{1}+b_{1}+a_{2}+b_{2}, \\
b \geq a_{1}+b_{2}, \quad b \geq a_{2}+b_{1}, \\
b \leq m(\widetilde{M}) .
\end{array}\right.
$$

In other words, the non-negativeness of interior measure, and the 
superadditivity of interior measure for disjoint sets and subadditivity of average measure for disjoint sets, and the inequalities on the third line of (6.2), and the monotone increasing property of exterior measure, form a complete set of conditions on the quantities $m_{i}(S)$ and $m_{e}(S)$ for $S=S_{1}, S_{2}$, and $S_{1} \cup S_{2}$, valid for every pair of disjoint sets $S_{1}, S_{2}$ contained in $\widetilde{M} \in \widetilde{\mathcal{M}}$.

In Theorem 8 above, consideration was given to partitionable measure spaces. If the measure space $(X, \mathcal{M}, m)$ is not partitionable, then from $\S 4$ there would be a set $M \in \mathcal{M}$ with $m(M)>0$ which is not a partitionable set, which means there is a set $B \subset M$, with $B \in \mathcal{M}$ and $m(B)>0$, for which there is no set $A \in \mathcal{M}$ satisfying (4.1). Thus, for this set $B$, every set $A \in \mathcal{M}$ with $A \subset B$ has $m(A)=m(B)$ or $m(A)=0$. The subset of $M$ consisting of all sets $A \in \mathcal{M}$ for which $A \subset B$, which could be designated by $\mathcal{M} \cap B$ and includes the set $B$, forms a countable ring, and the measure $m$ defined for $\mathcal{M} \cap B$ is countably additive. Thus $(B, \mathcal{M} \cap B, m)$ is also a measure space which is a 2 -valued measure space, having only the values 0 and $m(B)$ for the measures $m(A)$ of all sets $A \in(\mathcal{M} \cap B)$. This measure space is a part of $(X, \mathcal{M}, m)$.

If one considers all the sets $M \in \mathcal{M}$ for which $M \subset(X-B)$, then these form a countable ring $\mathcal{M} \cap(X-B)$ with a countably additive set function $m$, and so one has a measure space $(X-B, \mathcal{M} \cap(X-$ $B), m)$, which is also part of $(X, \mathcal{M}, m)$. And the original measure space $(X, \mathcal{M}, m)$ is a direct sum of the two measure spaces $(B, \mathcal{M} \cap$ $B, m)$ and $(X-B, \mathcal{M} \cap(X-B), m)$. Consideration can now be given to the measure space $(X-B, \mathcal{M} \cap(X-B), m)$, breaking off another 2 -valued measure space, if this is not a partitionable measure space, etc.. Suppose now that the original measure $(X, \mathcal{M}, m)$ satisfies the following countability condition for a measure space:

If $M_{\underline{\alpha}} \in \mathcal{M}$ with $m\left(M_{\underline{\alpha}}\right)>0$, are a collection of mutually disjoint sets $\in \mathcal{M}$ with positive measure, then there are at most a countable infinity of $M_{\underline{\alpha}}$.

If $(X, \mathcal{M}, m)$ satisfies this countability condition for a measure space, then one easily shows that $(X, \mathcal{M}, m)$ is a direct sum of a countable number of mutually disjoint 2 -valued measure spaces and of a partitionable measure space. Indicating this countable number of disjoint 2-valued measure spaces by the index $j$, where 
$j=1,2, \ldots$, to $n$ or to $\infty$, one has that

$$
\begin{aligned}
&(X, \mathcal{M}, m)=\bigoplus_{j}\left(B_{j}, \mathcal{M} \cap B_{j}, m\right) \\
& \oplus\left(X-\bigcup_{j} B_{j}, \mathcal{M} \cap\left(X-\bigcup_{j} B_{j}\right), m\right),
\end{aligned}
$$

where $B_{j} \in \mathcal{M}$ and $m\left(B_{j}\right)>0$, and $\left(B_{j}, \mathcal{M} \cap B_{j}, m\right)$ is a 2-valued measure space, and $B_{j_{1}} \cap B_{j_{2}}=\emptyset$ for all $j_{1}, j_{2}$ with $j_{1} \neq j_{2}$, and $\left(X-\bigcup_{j} B_{j}, \mathcal{M} \cap\left(X-\bigcup_{j} B_{j}\right), m\right)$ is a partitionable measure space (which may be a 0 -measure space in which every $M$ in $(\mathcal{M} \cap(X-$ $\left.\left.\bigcup_{j} B_{j}\right)\right)$ has $\left.m(M)=0\right)$. For any set $S$ in $X$, one has $S=\bigcup_{j}(S \cap$ $\left.B_{j}\right) \cup\left(S \cap\left(X-\bigcup_{j} B_{j}\right)\right)$, and by Lemma 1 ,

$$
m_{i}(S)=\sum_{j} m_{i}\left(S \cap B_{j}\right)+m_{i}\left(S \cap\left(X-\bigcup_{j} B_{j}\right)\right)
$$

and

$$
m_{e}(S)=\sum_{j} m_{e}\left(S \cap B_{j}\right)+m_{e}\left(S \cap\left(X-\bigcup_{j} B_{j}\right)\right) .
$$

For $m_{i}\left(S \cap B_{j}\right)$ and $m_{e}\left(S \cap B_{j}\right)$ one has only the two possible values 0 and $m\left(B_{j}\right)$, since $\left(B_{j}, \mathcal{M} \cap B_{j}, m\right)$ is 2-valued measure space.

Thus, in considering $m_{i}(S)$ and $m_{e}(S)$ for any set $S$, it is best to decompose the measure space as in (6.3), and treat the first part $\bigoplus_{j}\left(B_{j}, \mathcal{M} \cap B_{j}, m\right)$ separately from the second part $\left(X-\bigcup_{j} B_{j}, \mathcal{M} \cap\right.$ $\left.\left(X-\bigcup_{j} B_{j}\right), m\right)$ which is a partitionable measure space. This latter partitionable measure space also satisfies the countability condition for a measure space. Furthermore, it is easily shown that: If $(X, \mathcal{M}, m)$ is a partitionable measure space and satisfies the countability condition for a measure space, than every set $M$ which $\in \mathcal{M}$ also $\in \widetilde{\mathcal{M}}$, so that $\widetilde{\mathcal{M}}=\mathcal{M}$. 
An example of a 2 -valued measure space $(X, \mathcal{M}, m)$ is one in which $\mathcal{M}$ consists of the two sets $\emptyset$ and $X$, and $m(\emptyset)=0$ and $m(X)>0$. Then for any set $S$ in $X$, other than $\emptyset$ and $X$, one has $m_{i}(S)=0$ and $m_{e}(S)=m(X)$. The set $X$ could have any number of points, including a finite number. Another example of a two-valued measure space $(X, \mathcal{M}, m)$ is one in which $X$ consists of an uncountable infinity of points, and $\mathcal{M}$ consists of all subsets of $X$, and $m(M)=\infty$ if $M$ contains an uncountable infinity of points while $m(M)=0$ if the number of points in $M$ is countable. For this 2-valued measure space, every set is measurable. Still another example of a two-valued measure space is to take any measure space $(X, \mathcal{M}, m)$ and form a new measure space $(X, \mathcal{M}, \hat{m})$ with the same $X$ and $\mathcal{M}$ but with a different $\hat{m}$, where $\hat{m}(M)=0$ if $m(M)=0$ and $\hat{m}(M)=\infty$ if $m(M)>0$.

Incidentally, in connection with Theorem 8 for disjoint sets in a given $\widetilde{M} \in \widetilde{\mathcal{M}}$, one need only have the basis condition (5.1) with the $\chi^{(f)}$ and $\chi^{(p)}$ for the measure space $(\widetilde{M}, \mathcal{M} \cap \widetilde{M}, m)$ instead of the containing measure space $(X, \mathcal{M}, m)$, if these are different. Note that the measure space $(\widetilde{M}, \mathcal{M} \cap \widetilde{M}, m)$ in which $S_{1}$ and $S_{2}$ lie does satisfy the countability condition for a measure space.

In the definition of a general measure space $(X, \mathcal{M}, m)$, it was not assumed that $X \in \mathcal{M}$. If $X \notin \mathcal{M}$, one can extend the measure space $(X, \mathcal{M}, m)$, if it is desired, to a measure space $\left(X, \mathcal{M}^{*}, m^{*}\right)$ in which the extended countable ring $\mathcal{M}^{*}$ includes the set $X$ as well as all of $\mathcal{M}$, and the extended measure function $m^{*}$ coincides with $m$ over all of $\mathcal{M}$. The smallest such countable ring $\mathcal{M}^{*}$ is:

\section{(6.4) $\mathcal{M}^{*}=\mathcal{M}$ and the sets $\quad X-M \quad$ for all $\quad M \in \mathcal{M}$.}

Indeed, one easily shows that $\mathcal{M}^{*}$ in (6.4) is a countable ring. Note that the two parts of $\mathcal{M}^{*}$ in (6.4) have no sets in common, because $X-M_{1} \neq M_{2}$ for all $M_{1} \in \mathcal{M}, M_{2} \in \mathcal{M}$ since $X \notin \mathcal{M}$. Define the measure function $m^{*}$ by:

$$
\left\{\begin{array}{lll}
m^{*}(M)=m(M) & \text { for all } & M \in \mathcal{M} \\
m^{*}(X-M)=m_{i}(X-M) & \text { for all } & M \in \mathcal{M}
\end{array}\right.
$$

One can show that $m^{*}$ is countably additive for disjoint sets in $\mathcal{M}^{*}$. This uses $\left(X-M_{1}\right) \cap\left(X-M_{2}\right)=X-\left(M_{1} \cup M_{2}\right) \neq \emptyset$, so that two 
sets of the form $X-M$ are not disjoint. Thus, $\left(X, \mathcal{M}^{*}, m^{*}\right)$ is a measure space, an extension of $(X, \mathcal{M}, m)$.

In the extended measure space $\left(X, \mathcal{M}^{*}, m^{*}\right)$, any set $S$ has an interior measure $m_{i}^{*}(S)$ and an exterior measure $m_{e}^{*}(S)$. One can prove that:

$$
\begin{cases}m_{i}^{*}(S)=m_{i}(S) & \text { for all } S, \text { and } \\ m_{e}^{*}(S)=m_{e}(S) & \text { for all } S \text { such that } \quad S \subset M \in \mathcal{M}\end{cases}
$$

The measure space $\left(X, \mathcal{M}^{*}, m^{*}\right)$ is thus not only an extension of $(X, \mathcal{M}, m)$ but it also gives the same interior measure for every set $S$ in $X$ and it is an extension of the exterior measure applicable now to every set $S$ in $X$.

Concerning the countable ring $\widetilde{\mathcal{M}}$ defined in (2.10) and (2.8), one obtains a similar countable ring $\widetilde{\mathcal{M}}^{*}$ in the measure space $\left(X, \widetilde{\mathcal{M}}^{*}, m^{*}\right)$, and this includes $\widetilde{\mathcal{M}}$. Also, it is easy to show that if $(X, \mathcal{M}, m)$ is a partitionable measure space, so is $\left(X, \mathcal{M}^{*}, m^{*}\right)$ a partitionable measure space. And the measure spaces $(X, \mathcal{M}, m)$ and $\left(X, \mathcal{M}^{*}, m^{*}\right)$ have the same value for $\chi^{(f)}$, and the same $\chi^{(p)}$. And if $(X, \mathcal{M}, m)$ satisfies the countability condition for a measure space, so does $\left(X, \mathcal{M}^{*}, m^{*}\right)$.

It might be desirable to have a complete measure space, i.e. one in which every subset of a set of measure 0 is also measurable and has measure 0 . The measure space $\left(X, \mathcal{M}^{*}, m^{*}\right)$, if it isn't complete, can be extended to a complete measure space by the usual process of completion. The above extensions can be made beforehand, if it is desired, and have $X$ as measurable also. (Incidentally, if there are points of $X$ not contained in any $M \in \mathcal{M}$, one can limit consideration to the point set $\bigcup M$, and replace $X$ in the above by $\bigcup_{M \in \mathcal{M}} M$, or consider $\bigcup_{M \in \mathcal{M}} M$ as $X$.)

If one has any extension $\left(X, \mathcal{M}^{*}, \hat{m}\right)$ of $(X, \mathcal{M}, m)$ to the countable ring $\mathcal{M}^{*}$, one can prove that $\hat{m}$ is given by

$$
\begin{array}{r}
\hat{m}=m(M), \quad \hat{m}(X-M)=m_{i}(X-M)+\hat{c} \\
\text { for all } \quad M \in \mathcal{M},
\end{array}
$$

where $\hat{c}$ is a non-negative constant, $\hat{c} \geq 0$. Indeed, for any $\hat{c} \geq 0$, (6.7) does furnish a countably additive measure function $\hat{m}$. If there 
is a set $M_{1} \in \mathcal{M}$ for which $m_{i}\left(X-M_{1}\right)<\infty$, in which case there is a set $M_{2} \in \mathcal{M}$ for which $m_{i}\left(X-M_{2}\right)=0$, then for $\hat{c}>0$ the extension $\left(X, \mathcal{M}^{*}, \hat{m}\right)$ is different from $\left(X, \mathcal{M}^{*}, m^{*}\right)$. It might be desired to have such an extension, to represent some unknown measure. But note that $\hat{m}_{i}\left(X-M_{1}\right)=\hat{m}\left(X-M_{1}\right)=m_{i}(X-M)+\hat{c}>m_{i}\left(X-M_{1}\right)$, and $\hat{m}_{i}$ does not agree with $m_{i}$ for all sets $S$, as in (6.6). Thus, the extension $\left(X, \mathcal{M}^{*}, m^{*}\right)$ is the only extension of $(X, \mathcal{M}, m)$ to the countably ring $\mathcal{M}^{*}$ which satisfies (6.6).

It should be stated, in closing $\S 6$, that in all of the present article, and also in the author's articles [1] and [2], no hypothesis is made concerning the continuum $2^{\chi_{0}}$; the continuum hypothesis of set theory, which is that $2^{\chi_{0}}=\chi_{1}$, is not used. Use has been made of transfinite induction and of the axiom of choice.

7. Disjoint Sets in a Set $S$. In all of the preceding, consideration was given to disjoint sets $S_{1}, S_{2}$ contained in a set $\widetilde{M} \in \widetilde{\mathcal{M}}$. Now, consider disjoint sets $S_{1}, S_{2}$ contained in any set $S$. First, for any set $S$ contained in $M \in \mathcal{M}$, there is $m_{i}(S)$ and $m_{e}(S)$ for which $0 \leq m_{i}(S) \leq m_{e}(S)$, and the sets $A$ and $B$ in (2.3) and (2.6) will be chosen more carefully in the following lemma.

Lemma 6. Suppose that $S$ is a set in a measure space $(X, \mathcal{M}, m)$ and $S \subset \widetilde{M} \in \widetilde{\mathcal{M}}$, where $\widetilde{\mathcal{M}}$ is defined in (2.10) and (2.8). Then there are sets $K \in \widetilde{\mathcal{M}}$ and $L \in \widetilde{\mathcal{M}}$ such that

$$
\begin{gathered}
K \subset S \subset L \subset \widetilde{M}, \text { with } \\
m(K)=m_{i}(S), \quad m(L)=m_{e}(S), \\
\begin{cases}m_{i}(S-K)=0, & m_{i}(L-S)=0, \\
m_{e}(S-K)=m(L-K), & m_{e}(L-S)=m(L-K) .\end{cases}
\end{gathered}
$$

Further, considering $M \cap S$ for any $M \in \mathcal{M}$, and using $K, L$ satisfying (7.1) and (7.2), then (7.1) and (7.2) also hold with $M \cap K, M \cap$ $S, M \cap L$ in place of $K, S, L$ respectively.

Proof. Suppose first that $m_{e}(S)<\infty$. Then sets $A$ and $B$ in (2.3) and (2.6) can be selected as the sets $K$ and $L$ of Lemma 6 , and 
(7.1) is satisfied. Let $C_{1}$ by any set $\in \mathcal{M}$ with $C_{1} \subset(S-K)$. Then $C_{1} \cap K=\emptyset$ and $\left(C_{1} \cup K\right) \subset S$, so that $m\left(C_{1}\right)+m(K)=m\left(C_{1} \cup K\right) \leq$ $m_{i}(S)=m(K)$. Transposing $m(K)$ since $m(K)<\infty$ shows that $m\left(C_{1}\right) \leq 0$ and therefore $m\left(C_{1}\right)=0$, and so $m_{i}(S-K)=0$. Let $C_{2}$ be any set $\in \mathcal{M}$ with $C_{2} \subset(L-S)$. Then $C_{2} \cap S=\emptyset$ so that $S \subset\left(L-C_{2}\right)$, and $m(L)=m_{e}(S) \leq m\left(L-C_{2}\right)=m(L)-m\left(C_{2}\right)$, which gives $m\left(C_{2}\right) \leq 0$ since $m(L)<\infty$ and therefore $m\left(C_{2}\right)=0$, and so $m_{i}(L-S)=0$. Thus, the first line of (7.2) is established. Also, $(L-K)=(L-S) \cup(S-K)$ and $(L-S) \cap(S-K)=\emptyset$, so that by Lemma 2 ,

$$
m_{i}(L-S)+m_{e}(S-K)=m_{e}(L-S)+m_{i}(S-K)=m(L-K) .
$$

This gives, using the first line of (7.2), that $m_{e}(S-K)=m_{e}(L-$ $S)=m(L-K)$. Thus, (7.1) and (7.2) are established for $m_{e}(S)<$ $\infty$. Note, when $m_{e}(S)<\infty$, that (7.2) followed from (7.1) in the above proof.

Consider now the case that $m_{e}(S)=\infty$, so that $m(\widetilde{M})=\infty$. Write $\widetilde{M}=\bigcup_{j=1}^{\infty} M^{j}$ as in (2.9), where the $M^{j}$ are mutually disjoint and $M^{j} \in \widetilde{\mathcal{M}}$ and $m\left(M^{j}\right)<\infty$ for every $j$. Then $S=\bigcup_{j=1}^{\infty}\left(M^{j} \cap S\right)$, and for the set $M^{j} \cap S$ there is by the preceding paragraph sets $K^{j}$ and $L^{j}$, both $\in \widetilde{\mathcal{M}}$, with $K^{j} \subset\left(M^{j} \cap S\right) \subset L^{j} \subset M^{j}$, and formulas (7.1) and (7.2) hold with $K^{j}, M^{j} \cap S, L^{j}$ replacing $K, S, L$ respectively. Now, place $K=\bigcup_{j=1}^{\infty} K^{j}, L=\bigcup_{j=1}^{\infty} L^{j}$. Then $K \subset S \subset$ $L \subset \widetilde{M}$, and since the $M^{j}$ are mutually disjoint, so are the $K^{j}$, and also so are the $L^{j}$. One has

$$
m(K)=\sum_{j=1}^{\infty} m\left(K^{j}\right)=\sum_{j=1}^{\infty} m_{i}\left(M^{j} \cap S\right)=m_{i}(S)
$$

by Lemma 1 , formula $(2.7)$, since $\left(X-\bigcup_{j=1}^{\infty} M^{j}\right) \cap S=\emptyset$, and also

$$
m(L)=\sum_{j=1}^{\infty} m\left(L^{j}\right)=\sum_{j=1}^{\infty} m_{e}\left(M^{j} \cap S\right)=m_{e}(S)
$$


by Lemma 1, (2.7) again. So, (7.1) is established.

Also,

$$
(S-K)=\bigcup_{j=1}^{\infty}\left(M^{j} \cap(S-K)\right)=\bigcup_{j=1}^{\infty}\left(\left(M^{j} \cap S\right)-K^{j}\right)
$$

since $M^{j} \cap K=K^{j}$, and then by Lemma 1, (7.1) and (7.2) for finite $m_{e}$,

$$
m_{i}(S-K)=\sum_{j=1}^{\infty} m_{i}\left(\left(M^{j} \cap S\right)-K^{j}\right)=\sum_{j=1}^{\infty} 0=0,
$$

$m_{e}(S-K)=\sum_{j=1}^{\infty} m_{e}\left(\left(M^{j} \cap S\right)-K^{j}\right)=\sum_{j=1}^{\infty} m\left(L^{j}-K^{j}\right)=m(L-K)$ since $L-K=\bigcup_{j=1}^{\infty}\left(L^{j}-K^{j}\right)$. Likewise,

$$
L-S=\bigcup_{j=1}^{\infty}\left(M^{j} \cap(L-S)\right)=\bigcup_{j=1}^{\infty}\left(L^{j}-\left(M^{j} \cap S\right)\right)
$$

since $M^{j} \cap L=L^{j}$, and then by Lemma $1,(7.1)$ and (7.2) for finite $m_{e}$,

$$
m_{i}(L-S)=\sum_{j=1}^{\infty} m_{i}\left(L^{j}-\left(M^{j} \cap S\right)\right)=\sum_{j=1}^{\infty} 0=0
$$

$m_{e}(L-S)=\sum_{j=1}^{\infty} m_{e}\left(L^{j}-\left(M^{j} \cap S\right)\right)=\sum_{j=1}^{\infty} m\left(L^{j}-K^{j}\right)=m(L-K)$.

Formulas (7.2) are established.

Concerning the last sentence of Lemma 6, one has that $m_{i}((M \cap S)-(M \cap K))=0 \quad$ and $\quad m_{i}((M \cap L)-(M \cap S))=0$

which is the first line of (7.2) with $M \cap K, M \cap S, M \cap L$ in place of $K, S, L$ respectively, since $(M \cap S)-(M \cap K)=M \cap(S-K) \subset$ $(S-K)$ and $m_{i}(S-K)=0$, and likewise $(M \cap L)-(M \cap S)=$ $M \cap(L-S) \subset(L-S)$ and $m_{i}(L-S)=0$. By Lemma 1 ,

$$
\begin{aligned}
m_{i}(M \cap S) & =m_{i}(M \cap K)+m_{i}((M \cap S)-(M \cap K)) \\
& =m_{i}(M \cap K)+.0=m(M \cap K),
\end{aligned}
$$


and since $(M \cap L)=(M \cap S) \cup(M \cap(L-S))$, Lemma 2 gives $m(M \cap L)=m_{e}(M \cap S)+m_{i}(M \cap(L-S))=m_{e}(M \cap S)+0=m_{e}(M \cap S) ;$ thus, (7.1) holds with $M \cap K, M \cap S, M \cap L$ in place of $K, S, L$. Also, $(M \cap L)-(M \cap K)=((M \cap L)-(M \cap S)) \cup((M \cap S)-(M \cap K))$, so that by Lemma 2 ,

$$
\begin{gathered}
m((M \cap L)-(M \cap K)) \\
=m_{e}((M \cap L)-(M \cap S))+m_{i}((M \cap S)-(M \cap K)) \\
=m_{e}((M \cap L)-(M \cap S))+0=m_{e}((M \cap L)-(M \cap S)), \quad \text { and } \\
m((M \cap L)-(M \cap K)) \\
=m_{i}((M \cap L)-(M \cap S))+m_{e}((M \cap S)-(M \cap K)) \\
=0+m_{e}((M \cap S)-(M \cap K))=m_{e}((M \cap S)-(M \cap K)) .
\end{gathered}
$$

So, the second line of (7.2) holds with $M \cap K, M \cap S, M \cap L$ in place of $K, S, L$; and also the first line of (7.2) holds. The last sentence of Lemma 6 is therefore established, and Lemma 6 is proved.

Now consider disjoint sets $S_{1}, S_{2}$ contained in a set $S$ where $S \subset$ $\widetilde{M} \in \widetilde{\mathcal{M}}$ :

(7.3) $\quad S_{1} \cap S_{2}=\emptyset,\left(S_{1} \cup S_{2}\right) \subset S \quad$ where $\quad S \subset \widetilde{M} \in \widetilde{\mathcal{M}}$.

By Theorem 4 and (3.19) and (3.20), there are the six non-negative quantities $a_{1}, a_{2}, d_{i}, h, g_{1}, g_{2}$ satisfying (3.21) and (3.22). The converse, as in Theorem 8 , will now be discussed. Note that there is the formula

$$
a_{1}+a_{2}+d_{i} \leq m_{i}(S)
$$

as well as $a_{1}+a_{2}+d_{i}+h+g_{1}+g_{2} \leq m_{e}(S)$.

As in $\S 5$, consider the cardinal number of points in a set $S$ for which $m_{e}(S)>0$, and let $\chi^{(\bar{p})}$ denote the least cardinal number of points in such sets $S$, among all sets $S$ with $m_{e}(S)>0$. This is analogous to $\S 5$, in defining $\chi^{(p)}$, and note that $\chi^{(\bar{p})} \leq \chi^{(p)}$. The measure space $(X, M, m)$ will be said to satisfy the basis condition for partially measurable sets if

$$
\chi^{(\bar{p})} \geq \chi^{(f)}
$$


where $\chi^{(f)}$ is defined in $\S 5$. And also suppose as in (5.2) the following.

(7.6) $\left\{\begin{array}{l}\text { Every set } S \text { with } m_{e}(S)>0 \text { contains an } \\ \text { infinity of points, besides the first sentence of (5.2). }\end{array}\right.$

Let $\underline{w}^{(\bar{p})}$ denote the smallest ordinal number of cardinality $\chi^{(\bar{p})}$. There is the Theorem 9 below, analogous to Theorems 6 and 7 .

THEOREM 9. Suppose that $(X, \mathcal{M}, m)$ is a measure space satisfying the basis condition (7.5) for partially measurable sets, and also (7.6). Consider a set $S$ in $X$ with $m_{e}(S)>0$ and $S \subset \widetilde{M}$ where $\widetilde{M} \in \widetilde{\mathcal{M}}$, and let $L$ be a set $\in \widetilde{\mathcal{M}}$ with

$$
S \subset L \quad \text { and } \quad m(L)=m_{e}(S), \quad m_{i}(L-S)=0,
$$

as in Lemma 6. Then for any cardinal number $\leq \chi^{(\bar{p})}$ and $\geq 2$, there are that number of mutually disjoint sets $Z_{\beta}$ contained in $S$, where $\underline{\beta}$ runs over all non-negative ordinal numbers of cardinality $<$ that cardinal number, such that

$$
\begin{gathered}
S=\bigcup_{\underline{\beta}} Z_{\underline{\beta}} \text { and } m_{i}\left(Z_{\underline{\beta}}\right)=0, \\
m_{i}\left(L-Z_{\underline{\beta}}\right)=0 \text { for all } \underline{\beta} .
\end{gathered}
$$

Proof. Let $\mathcal{C}^{(f)}$ be a collection of sets $C_{\eta} \in \mathcal{M}$ with $m\left(C_{\eta}\right)>0$, which form a measure basis for $(X, \mathcal{M}, m)$, there being $\chi^{(f)}$ number of sets $C_{\eta}$ in the collection $\mathcal{C}^{(f)}$. Consider all those sets $C_{\eta}$ in $\mathcal{C}^{(f)}$ for which $m_{e}\left(C_{\underline{\eta}} \cap S\right)>0$; the totality of all such $C_{\underline{\eta}}$ forms a collection $\mathcal{C}^{(\bar{f})}$, where $\overline{\mathcal{C}}^{(\bar{f})}$ is a part of $\mathcal{C}^{(f)}$. Let $\chi^{(\bar{f})}$ denote the cardinal number of sets $C_{\underline{\eta}}$ in $\mathcal{C}^{(\bar{f})}$, so that $\chi^{(\bar{f})} \leq \chi^{(f)}$, and let $\underline{w}^{(\bar{f})}$ be the smallest ordinal number of cardinality $\chi^{(\bar{f})}$. The totality of all the $C_{\underline{\eta}}$ in $\mathcal{C}^{(\bar{f})}$ can be written as $C_{\underline{\eta}_{\alpha}}$, where $\underline{\alpha}$ runs over all non-negative ordinal numbers $<\underline{w}^{(\bar{f})}$, and designate the $C_{\underline{\eta}_{\alpha}}$ more simply as $\bar{C}_{\underline{\alpha}}$. Thus, the collection $\mathcal{C}^{(\bar{f})}$ consists of the sets $\frac{\underline{\alpha}}{\bar{C}_{\underline{\alpha}}}$ with

$m_{e}\left(\overline{C_{\underline{\alpha}}} \cap S\right)>0, \quad$ for all non-negative ordinals $\quad \underline{\alpha}<\underline{w}^{(\bar{f})}$ 
and the measure basis collection $\mathcal{C}^{(f)}$ consists of $\mathcal{C}^{(\bar{f})}$ and also all $C_{\underline{\eta}}$ for which $m_{e}\left(C_{\underline{\eta}} \cap S\right)=0$.

Now, proceed as in the proof of Theorem 6 , with $\chi^{(\bar{p})}$ and $\underline{w}^{(\bar{p})}$ (where $\underline{w}^{(\bar{p})}$ is the smallest ordinal number of cardinality $\chi^{(\bar{p})}$ ), and $\chi^{(\bar{f})}$ and $\underline{w}^{(\bar{f})}$, replacing $\chi^{(p)}$ and $\underline{w}^{(p)}$, and $\chi^{(f)}$ and $\underline{w}^{(f)}$, respectively. As in Lemma 5 , but with $\overline{C_{\underline{\alpha}}} \cap S$ replacing $C_{\underline{\alpha}}$, one proves that there are points $y_{\underline{\alpha}}$ and $\left.x_{(} \underline{\alpha}, \underline{\beta}\right)$ in $S$, for all ordinals $\underline{\alpha}<\underline{w}^{(\bar{f})}$ and all ordinals $\underline{\beta}<\underline{w}^{(\bar{p})}$, which are all different points, and

$$
\begin{gathered}
\left.y_{\underline{\alpha}} \in\left(\overline{C_{\underline{\alpha}}} \cap S\right), x_{(\underline{\alpha}}, \underline{\beta}\right) \in\left(\overline{C_{\underline{\alpha}}} \cap S\right), \\
\text { for all } \underline{\alpha}<\underline{w}^{(\bar{f})} \text { and all } \underline{\beta}<\underline{w}^{(\bar{p})} .
\end{gathered}
$$

The proof of this is the same as the proof of Lemma 5 , with $\overline{C_{\underline{\alpha}}} \cap S$ replacing $C_{\underline{\alpha}}$ in the proof, noting that $m_{e}\left(\overline{C_{\underline{\alpha}}} \cap S\right)>0$ so that $\overline{C_{\underline{\alpha}}} \cap S$ contains at least $\chi^{(\bar{p})}$ points, and $\chi^{(\bar{p})} \geq \chi^{(f)} \geq \chi^{(\bar{f})}$ by $(7.5)$.

Proceeding as in the proof of Theorem 6 after the proof of Lemma 5, define the set

$$
\begin{array}{r}
Z_{\underline{\beta}} \quad \text { as in }(5.9) \text { for } \quad 0<\underline{\beta}<\underline{w}^{(\bar{p})}, \\
\text { and } Z_{0}=S-\bigcup_{1 \leq \underline{\beta}^{<} \underline{w}^{(\bar{p})}} Z_{\underline{\beta}},
\end{array}
$$

so that $S=\bigcup_{\underline{\beta}} Z_{\underline{\beta}}$ as in (7.7). Let $M$ be a set $\in \mathcal{M}$ with $M \subset Z_{\underline{\beta}}$. If $m(M)>0$, there is a set $C_{\underline{\eta}} \in \mathcal{C}^{(f)}$ with $m\left(C_{\underline{\eta}}\right)>0$ and $M \supset C_{\underline{\eta}}$, so that $C_{\underline{\eta}} \subset Z_{\underline{\beta}} \subset S$. Since $C_{\underline{\eta}} \cap S=C_{\underline{\eta}}, C_{\underline{\eta}}$ is a $\bar{C}_{\underline{\alpha}} \in \mathcal{C}^{(\bar{f})}$, and the same contradiction as in the proof of Theorem 6 shows that $m(M)=0$. Thus, $m_{i}\left(Z_{\underline{\beta}}\right)=0$ as in (7.7).

Concerning the set $L-Z_{\underline{\beta}}$, let $M$ be a set $\in \mathcal{M}$ with $M \subset\left(L-Z_{\underline{\beta}}\right)$. If $m(M)>0$, there is a set $C_{\underline{\eta}} \in \mathcal{C}^{(f)}$ with $m\left(C_{\underline{\eta}}\right)>0$ and $M \supset \bar{C}_{\underline{\eta}}$, so that $C_{\underline{\eta}} \subset\left(L-Z_{\underline{\beta}}\right)$. If $m_{e}\left(C_{\underline{\eta}} \cap S\right)>0$, then $C_{\underline{\eta}}$ is a $\bar{C}_{\underline{\alpha}} \in \mathcal{C}^{(\bar{f})}$, and $\bar{C}_{\underline{\alpha}} \subset\left(L-Z_{\underline{\beta}}\right)$ with $m\left(\bar{C}_{\underline{\alpha}}\right)>0$. But for $\underline{\beta}>0, x(\underline{\alpha}, \underline{\beta}) \in \bar{C}_{\underline{\alpha}}$ and $\left.x_{(\underline{\alpha}}, \underline{\beta}\right) \notin\left(L-Z_{\underline{\beta}}\right)$ by $(7.8)$ and $(7.9)$ and $(5.9)$, so that $\bar{C}_{\underline{\alpha}} \not \subset$ $\left(L-Z_{\underline{\beta}}\right)$; and for $\underline{\beta}=0, y_{\underline{\alpha}} \in \bar{C}_{\underline{\alpha}}$ by (7.8) but $y_{\underline{\alpha}} \in Z_{0}$ by (7.9) and (5.9), so that $\bar{C}_{\underline{\alpha}} \not \subset\left(L-Z_{0}\right)$. These contradictions show that $m_{e}\left(C_{\underline{\eta}} \cap S\right)=0$. Let $Q$ be a set $\in \mathcal{M}$ with $C_{\underline{\eta}} \supset Q \supset\left(C_{\underline{\eta}} \cap S\right)$ and $\bar{m}(Q)=m_{e}\left(C_{\underline{\eta}} \cap S\right)=0$. Then $m\left(C_{\underline{\eta}}-Q\right)^{\underline{2}}=m\left(C_{\eta}\right)>\overline{0}$, and 
$\left(C_{\eta}-Q\right) \cap S=\emptyset ;$ and $\left(C_{\eta}-Q\right) \subset C_{\eta} \subset\left(L-Z_{\beta}\right) \subset L$, so that $\left(C_{\underline{\eta}}^{-}-Q\right) \subset(L-S)$. Since $\bar{m}_{i}(L-S)=\overline{0}$, one has $m\left(C_{\underline{\eta}}-Q\right)=0$. This contradicts that $m\left(C_{\eta}-Q\right)>0$. Therefore the assumption that $m(M)>0$ is false, and so $m(M)=0$. This is for every $M \in \mathcal{M}$ with $M \subset\left(L-Z_{\beta}\right)$, so that $m_{i}\left(L-Z_{\beta}\right)=0$. Thus, (7.7) is established, and Theorem 9 is proved for a cardinal number $=\chi^{(\bar{p})}$.

Theorem 9 for a cardinal number $<\chi^{(\bar{p})}$ and $\geq 2$ is proved as in the proof of Theorem 7 , using $L-Z_{1}, L-Z_{0}$, and $\left(L-\widehat{Z}_{0}\right)$ in place of $X-Z_{1}, X-Z_{0}$, and $\left(X-\widehat{Z}_{0}\right)$ respectively. The proof of Theorem 9 is completed.

Incidentally, Theorem 9 shows that even if $m_{i}(S)=0, S$ can be decomposed as in (7.7). For example, any particular $Z_{\beta}$ in (7.7) can itself be decomposed as in (7.7).

Now, there is Theorem 4, and analogous to Theorem 8 there is the following theorem.

THEOREM 10. Suppose that $(X, \mathcal{M}, m)$ is a partitionable measure space (this is defined in the first paragraph of $\S$ 4) satisfying the basis condition (7.5) for partially measurable sets. Let $S$ be a set for which

$$
m_{e}(S)>0 \quad \text { and } \quad S \subset \widetilde{M} \text { for some } \widetilde{M} \in \widetilde{\mathcal{M}}
$$

Then, given any six non-negative real numbers or $\infty$, namely

$$
\begin{gathered}
a_{1}, a_{2}, d_{i}, h, g_{1}, g_{2} \quad \text { all } \geq 0, \quad \text { satisfying } \\
\left\{\begin{array}{l}
a_{1}+a_{2}+d_{i}+h+g_{1}+g_{2} \leq m_{e}(S), \quad \text { and } \\
a_{1}+a_{2}+d_{i} \leq m_{i}(S),
\end{array}\right.
\end{gathered}
$$

there is a pair of disjoint sets $S_{1}, S_{2}$ contained in $S$ such that

(7.11) $\left\{\begin{array}{l}m_{i}\left(S_{1}\right)=a_{1}, \quad m_{i}\left(S_{2}\right)=a_{2}, \quad d_{i}\left(S_{1}, S_{2}\right)=d_{i}, \\ g_{1}\left(S_{1}, S_{2}\right)=g_{1}, \quad g_{2}\left(S_{1}, S_{2}\right)=g_{2}, \quad h\left(S_{1}, S_{2}\right)=h .\end{array}\right.$

And formulas (6.1) hold.

Proof. Concerning the set $S$ one has Lemma 6, including the sets $K$ and $L$ in $\widetilde{\mathcal{M}}$ satisfying (7.1) and (7.2). Consider first the case 
that $m_{e}(S)<\infty$, so that $a_{1}, a_{2}, d_{i}, h, g_{1}, g_{2}$ are all finite by (7.10). Select three mutually disjoint sets

$$
\left\{\begin{array}{l}
A_{1}, A_{2}, \text { and } D_{i}, \text { mutually disjoint and } \subset K \\
\text { and } \in \widetilde{\mathcal{M}}, \text { with } \\
m\left(A_{1}\right)=a_{1}, \quad m\left(A_{2}\right)=a_{2}, \quad m\left(D_{i}\right)=d_{i} .
\end{array}\right.
$$

This is possible by the second formula of (7.10) and Theorem 5 and the paragraph preceding Lemma 4 in $§ 4$. Place

$$
\left\{\begin{array}{l}
\bar{K}=K-\left(A_{1} \cup A_{2} \cup D_{i}\right), \\
\bar{L}=L-\left(A_{1} \cup A_{2} \cup D_{i}\right), \quad \text { and } \\
\bar{S}=S-\left(A_{1} \cup A_{2} \cup D_{i}\right),
\end{array}\right.
$$

so that $\bar{K} \subset \bar{S} \subset \bar{L} \subset \widetilde{M}$. By Lemma 1 ,

$$
\begin{gathered}
m_{i}(\bar{S})=m_{i}(S)-\left(a_{1}+a_{2}+d_{i}\right)=m(K)-\left(a_{1}+a_{2}+d_{i}\right)=m(\bar{K}), \quad \text { and } \\
m_{e}(\bar{S})=m_{e}(S)-\left(a_{1}+a_{2}+d_{i}\right)=m(L)-\left(a_{1}+a_{2}+d_{i}\right)=m(\bar{L}), \\
\text { and } \bar{S}-\bar{K}=S-K, \quad \bar{L}-\bar{S}=L-S, \text { and } \bar{L}-\bar{K}=L-K
\end{gathered}
$$

These state that the sets $\bar{K}$ and $\bar{L}$ are the $K$ and $L$ of Lemma 6 for the set $\bar{S}$, satisfying (7.1). And (7.2) is satisfied for $\bar{S}$ and $\bar{K}, \bar{L}$ because (7.2) followed from (7.1) in the proof of Lemma 6. Since

$$
m(\bar{L})=m_{e}(S)-\left(a_{1}+a_{2}+d_{i}\right) \geq h+g_{1}+g_{2},
$$

by the first of (7.10), select three mutually disjoint sets

$$
\left\{\begin{array}{l}
H, G_{1}, \text { and } G_{2}, \text { mutually disjoint } \\
\text { and } \subset \bar{L} \text { and } \in \widetilde{\mathcal{M}}, \text { with } \\
m(H)=h, \quad m\left(G_{1}\right)=g_{1}, \quad m\left(G_{2}\right)=g_{2} .
\end{array}\right.
$$

This is possible by (7.14), and Theorem 5 and the paragraph preceding Lemma 4 in $\S 4$.

Now, apply Theorem 9 for the set $\bar{S}$. If $m_{e}(\bar{S})>0$, then since $\bar{S} \subset \widetilde{M} \subset \widetilde{\mathcal{M}}$, every such $\bar{S}$ contains an uncountable infinity of points. For, a point of $\bar{S}$ is contained in a set $M_{0} \subset \widetilde{M}$ with $M_{0} \in$ $\mathcal{M}$ and $m\left(M_{0}\right)=0$, by Lemma 4 . If there are only a countable 
number of points in $\bar{S}$, then $\bar{S}$ would be contained in a countable union of such sets, which is a set in $\mathcal{M}$ of $m$-measure 0 , so that $m_{e}(\bar{S})=0$, contrary to $m_{e}(\bar{S})>0$. Thus, the condition (7.6) is satisfied, and apply Theorem 9 for 3 sets. There are three mutually disjoint sets $Z_{1}, Z_{2}$, and $Z_{3}$ in $\bar{S}$, with $\bar{S}=Z_{1} \cup Z_{2} \cup Z_{3}$, satisfying (7.7) using $\bar{S}$ and $\bar{L}$ for these three sets. If $m_{e}(\bar{S})=0$, choose any three mutually disjoint sets in $\bar{S}$, possibly including $\emptyset$, whose union is $\bar{S}$, for $Z_{1}, Z_{2}, Z_{3}$. Also, by Theorem 9 for 2 sets, there are two disjoint sets $Z$ and $D_{i}-Z$ in $D_{i}$ with $m_{i}(Z)=0$ and $m\left(D_{i}-Z\right)=0$. Place

$$
\left\{\begin{array}{l}
S_{1}=A_{1} \cup Z \cup\left(H \cap Z_{1}\right) \cup\left(G_{1} \cap Z_{1}\right), \\
S_{2}=A_{2} \cup\left(D_{i}-Z\right) \cup\left(H \cap Z_{2}\right) \cup\left(G_{2} \cap Z_{2}\right), \\
\text { so that } S_{1} \cup S_{2}=A_{1} \cup A_{2} \cup D_{i} \\
\cup\left(H \cap\left(Z_{1} \cup Z_{2}\right)\right) \cup\left(G_{1} \cup Z_{1}\right) \cup\left(G_{2} \cap Z_{2}\right) .
\end{array}\right.
$$

The sets $A_{1}, A_{2}, D_{i}, H, G_{1}, G_{2}$ are mutually disjoint sets $\in \widetilde{\mathcal{M}}$, so that by Lemma 1 ,

$$
\text { (7.17) } \begin{cases}m_{i}\left(S_{1}\right) & =m\left(A_{1}\right)+0+0+0=m\left(A_{1}\right), \\ m_{i}\left(S_{2}\right) & =m\left(A_{2}\right)+0+0+0=m\left(A_{2}\right), \\ m_{i}\left(S_{1} \cup S_{2}\right)= & m\left(A_{1}\right)+m\left(A_{2}\right)+m\left(D_{i}\right)+0 \\ & +0+0=m\left(A_{1}\right)+m\left(A_{2}\right)+m\left(D_{i}\right),\end{cases}
$$

since $H \cap\left(Z_{1} \cup Z_{2}\right)=\left(H \cap\left(\bar{S}-Z_{3}\right)\right) \subset\left(\bar{S}-Z_{3}\right) \subset\left(\bar{L}-Z_{3}\right)$ and $m_{i}\left(\bar{L}-Z_{3}\right)=0$ by $(7.7)$ for $\bar{S}, \bar{L}$. Concerning exterior measures for ( 7.16 ), one has by Lemma 2

$$
m_{i}\left(H-\left(H \cap Z_{1}\right)\right)+m_{e}\left(H \cap Z_{1}\right)=m(H),
$$

and $H-\left(H \cap Z_{1}\right)=\left(H-Z_{1}\right) \subset\left(\bar{L}-Z_{1}\right)$ so that $m_{i}\left(H-\left(H \cap Z_{1}\right)\right)=0$ because $m_{i}\left(\bar{L}-Z_{1}\right)=0$. Therefore, $m_{e}\left(H \cap Z_{1}\right)=m(H)$. Likewise for the other terms in applying $m_{e}$ to (7.16), including $Z$ and $D_{i}-Z$ contained in $D_{i}$; and $m_{e}\left(H \cap\left(Z_{1} \cup Z_{2}\right)\right)=m(H)$ since $\left(H \cap Z_{1}\right) \subset$ $\left(H \cap\left(Z_{1} \cup Z_{2}\right)\right) \subset H$. There results, by Lemma 1:

$$
\begin{cases}m_{e}\left(S_{1}\right) & =m\left(A_{1}\right)+m\left(D_{i}\right)+m(H)+m\left(G_{1}\right), \\ m_{e}\left(S_{2}\right) & =m\left(A_{2}\right)+m\left(D_{i}\right)+m(H)+m\left(G_{2}\right), \\ m_{e}\left(S_{1} \cup S_{2}\right) & =m\left(A_{1}\right)+m\left(A_{2}\right)+m\left(D_{i}\right) \\ & \quad+m(H)+m\left(G_{1}\right)+m\left(G_{2}\right) .\end{cases}
$$


Concerning the functionals of the disjoint pair $S_{1}, S_{2}$ for the case that $m_{e}(S)<\infty$, the quantities $a_{1}, a_{2}, d_{i}, h, g_{1}, g_{2}$ are finite by (7.10). One has from (3.2), (7.17), and (7.12) that

$$
d_{i}\left(S_{1}, S_{2}\right)=m\left(D_{i}\right)=d_{i}
$$

Also, from (3.2), (7.17), (7.18) and (7.12), (7.15), one has the first two lines of $(7.20)$ :

$$
\left\{\begin{aligned}
g_{1}\left(S_{1}, S_{2}\right)= & \left(a_{1}+a_{2}+d_{i}+h+g_{1}+g_{2}\right)-a_{1} \\
& -\left(a_{2}+d_{i}+h+g_{2}\right)=g_{1}=m\left(G_{1}\right), \\
g_{2}\left(S_{1}, S_{2}\right)= & \left(a_{1}+a_{2}+d_{i}+h+g_{1}+g_{2}\right)-a_{2} \\
& -\left(a_{2}+d_{i}+h+g_{1}\right)=g_{2}=m\left(G_{2}\right), \\
\text { and } h\left(S_{1}, S_{2}\right)= & h=m(H) .
\end{aligned}\right.
$$

The third line of (7.20) follows similarly from (3.2), (7.17), (7.18) and (7.12), (7.15). Thus, the equations (7.11) and (6.1) are established, and Theorem 10 is proved for the case that $m_{e}(S)<\infty$.

If $m_{e}(S)=\infty$, then $m(\widetilde{M})=\infty$ since $S \subset \widetilde{M} \in \widetilde{\mathcal{M}}$, and express $\widetilde{M}$ as in (2.9): $\widetilde{M}=\bigcup_{j=1}^{\infty} M^{j}$ with $m\left(M^{j}\right)<\infty$ for all $j$ and the $M^{j}$ are mutually disjoint. Then $S=\widetilde{M} \cap S=\bigcup_{j=1}^{\infty}\left(M^{j} \cap S\right)$, and apply what has been proved above to the set $M^{j} \cap S$, using Lemma 6. By Lemma 6 with $M^{j} \cap K, M^{j} \cap S, M^{j} \cap L$ in place of $K, S, L$, one has by (7.1) that

$$
m\left(M^{j} \cap K\right)=m_{i}\left(M^{j} \cap S\right), \quad m\left(M^{j} \cap L\right)=m_{e}\left(M^{j} \cap S\right),
$$

and by (7.2),

$$
\begin{aligned}
& m_{i}\left(\left(M^{j} \cap S\right)-\left(M^{j} \cap K\right)\right)=0, \quad m_{i}\left(\left(M^{j} \cap L\right)-\left(M^{j} \cap S\right)\right)=0, \\
& m_{e}\left(\left(M^{j} \cap S\right)-\left(M^{j} \cap K\right)\right) \\
& \quad=m_{e}\left(\left(M^{j} \cap L\right)-\left(M^{j} \cap S\right)\right)=m\left(\left(M^{j} \cap L\right)-\left(M^{j} \cap K\right)\right) .
\end{aligned}
$$

Using formulas (7.19) and (7.20), there is obtained

$$
d_{i}\left(M^{j} \cap S_{1}, M^{j} \cap S_{2}\right)=m\left(M^{j} \cap D_{i}\right),
$$




$$
\begin{aligned}
g_{1}\left(M^{j} \cap S_{1}, M^{j} \cap S_{2}\right) & =m\left(M^{j} \cap G_{2}\right), \\
g_{2}\left(M^{j} \cap S_{1}, M^{j} \cap S_{2}\right) & =m\left(M^{j} \cap G_{2}\right), \\
h\left(M^{j} \cap S_{1}, M^{j} \cap S_{2}\right) & =m\left(M^{j} \cap H\right) .
\end{aligned}
$$

By Theorem 1, noting that $\left(X-\bigcup_{j=1}^{\infty} M^{j}\right) \cap\left(S_{1} \cup S_{2}\right)=\emptyset$ since $\left(S_{1} \cup S_{2}\right) \subset \widetilde{M}=\bigcup_{j=1}^{\infty} M^{j}$

$$
\left\{\begin{array}{l}
d_{i}\left(S_{1}, S_{2}\right)=\sum_{j=1}^{\infty} m\left(M^{j} \cap D_{i}\right)=m\left(D_{i}\right)=d_{i}, \\
g_{1}\left(S_{1}, S_{2}\right)=\sum_{j=1}^{\infty} m\left(M^{j} \cap G_{1}\right)=m\left(G_{1}\right)=g_{1}, \\
g_{2}\left(S_{1}, S_{2}\right)=\sum_{j=1}^{\infty} m\left(M^{j} \cap G_{2}\right)=m\left(G_{2}\right)=g_{2}, \\
h\left(S_{1}, S_{2}\right)=\sum_{j=1}^{\infty} m\left(M^{j} \cap H\right)=m(H)=h,
\end{array}\right.
$$

since all the sets involved are contained in $\widetilde{M}=\bigcup_{j=1}^{\infty} M^{j}$ using (7.12) and (7.15). Also, $m_{i}\left(S_{1}\right)=a_{1}, m_{i}\left(S_{2}\right)=a_{2}$ from (7.17). Theorem 10 is proved.

Theorem 10 is analogous to Theorem 8, but for two disjoint sets contained in any set $S \subset \widetilde{M} \in \widetilde{\mathcal{M}}$. (Note that in Theorems 10 and 8 , one could also have $m_{e}(S)=0$ or $m(\widetilde{M})=0$ by selecting the empty set $\emptyset$ for both $S_{1}$ and $S_{2}$.)

Incidentally, the proof of Theorem 10 above also gives a proof of Theorem 8. Theorem 8 is the case of Theorem 10 when $S=$ $\widetilde{M} \in \widetilde{\mathcal{M}}$. Then $m_{i}(S)=m_{e}(S)=m(\widetilde{M})$, and the second line of (7.10) is a consequence of the first line of (7.10), and also $K=S=$ $L=\widetilde{M}$ in the proof of Theorem 10. (Note that if one makes the extension of $(X, \mathcal{M}, m)$ to the measure space $\left(X, \mathcal{M}^{*}, m^{*}\right)$ as in $\S 6$, then the $\chi^{(\bar{p})}$ for $\left(X, \mathcal{M}^{*}, m^{*}\right)$ may possibly be smaller than the $\chi^{(\bar{p})}$ for $(X, \mathcal{M}, m)$.)

Consider Lebesgue or Borel measure on the real number line or in Euclidean $n$-space as the measure space. Condition (7.6) is satisfied, and if one assumes the continuum hypothesis of set theory, that 
$2^{\chi_{0}}=\chi_{1}$, then $\chi^{(\bar{p})}=\chi^{(f)}=2^{\chi_{0}}$ and (7.5) is satisfied. The measure space is also partitionable, so that the results of Theorems 9 and 10 hold for Lebesgue or Borel measure, if one assumes the continuum hypothesis of set theory.

If one does not assume the continuum hypothesis, there is an extension of Lebesgue measure, or it is Lebesgue measure itself, for which $\chi^{(\bar{p})}=\chi^{(f)}=2^{\chi_{0}}$. Namely, consider the collection $\widehat{\mathcal{M}}$ of all sets of the form $\left(L \cup R_{1}\right)-R_{2}$ where $L$ is Lebesgue measurable and $R_{1}, R_{2}$ are both sets of fewer than a continuum number $2^{\chi_{0}}$ of points, and place $\hat{m}\left(\left(L \cup R_{1}\right)-R_{2}\right)=m(L)$. One can show that $\widehat{\mathcal{M}}$ is a countable ring, and that $\hat{m}$ is uniquely defined in $\widehat{\mathcal{M}}$ and is countably additive, so that a measure space is formed. A set $R$ with fewer than $2^{\chi_{0}}$ points has $m_{i}(R)=0$, and I believe it is not known what $m_{e}(R)$ might be if one does not assume the continuum hypothesis; but $R \in \widehat{\mathcal{M}}$ and $\hat{m}(R)=0$ in the above measure space. For this measure space $\chi^{(\bar{p})}=2^{\chi_{0}}=\chi^{(\bar{f})}$ and the results of Theorems 9 and 10 hold. Also, it is reasonable to consider the measure space $(X, \widehat{\mathcal{M}}, \hat{m})$ since for a set $R$ with fewer than a continuum number $2^{\chi_{0}}$ of points, on the real number line or in Euclidean space, there are a continuum number of mutually disjoint translations of $R$. It is reasonable to consider $R$ to be of measure 0 .

\section{Bibliography}

[1] M.Shiffman, Measure-theoretic Properties of Arbitrary Point Sets, Proc. Nat. Acad. Sc., U.S.A.,67(1970), 302-304.

[2] M.Shiffman, Measure-theoretic Properties of Non-measurable Sets, Pacific J. Math. 138(1989), 357-389.

Received December 12,1991 and in revised form April 16, 1993.

California State University

HAYWARD, CA 94542 



\section{CONTENTS}

S. Abdulali, Conjugates of strongly equivariant maps .............. 207

C. Adams, Dehn filling hyperbolic 3-manifolds ...................... 217

S. Berman and B. Cox, Enveloping algebras and representations of toroidal Lie algebras.......................................... 239

B. Cox, Verma modules induced from nonstandard Borel subalgebras . 269

C. H. FitzGerald and C. R. Thomas, Some bounds on convex mappings in several complex variables..., ...................... 295

P. H. Loi, On the derived towers of certain inclusions of type $I I I_{\lambda}$ fac-

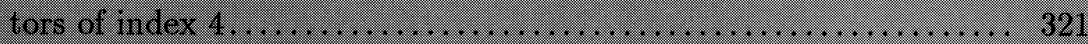

F. Morgan, Soap bubbles in $\mathbb{R}^{2}$ and in surfaces..................... 347

M. Shiffman, Partially measurable sets in measur spaces ............. 363 


\section{PACIFIC JOURNAL OF MATHEMATICS}

Volume $165 \quad$ No. $2 \quad$ October 1994

Conjugates of strongly equivariant maps

207

SALMAN ABDULALI

Dehn filling hyperbolic 3-manifolds

217

COLIN C. ADAMS

Enveloping algebras and representations of toroidal Lie algebras

239

STEPHEN BERMAN and BEN COX

Verma modules induced from nonstandard Borel subalgebras

269

BEN COX

Some bounds on convex mappings in several complex variables

295

CARL HANSON FitzGERALD and CAROLYN R. THOMAS

On the derived towers of certain inclusions of type $I I I_{\lambda}$ factors of

321 index 4

PHAN HUNG LOI

Soap bubbles in $\mathbb{R}^{2}$ and in surfaces

347

FRANK MORGAN

Partially measurable sets in measure spaces

MAX SHIFFMAN 